\title{
Light-Scattering Patterns from Stretched Films Having Fibrillar Structures in Relation to the Deformation Mechanism of a Random Assembly of Anisotropic Rods in Two Dimensions*
}

\author{
Norio Hayashi, ${ }^{* *}$ Yukinobu Murakami, Masahiko Moritani, ${ }^{* * *}$, \\ Takeji HaShimoto, and Hiromichi KaWAI*** \\ Department of Polymer Chemistry, Faculty of Engineering, \\ Kyoto University, Kyoto, Japan.
}

(Received October 4, 1972)

\begin{abstract}
Experimentally observed $H_{\mathrm{V}}$ and $V_{\mathrm{V}}$ light-scattering patterns from stretched films of collagen, poly(chlorotrifluoroethylene), polypropylene and polyethylene A-B type block copolymers, and hydroxypropylcellulose were theoretically explained in terms of a model based upon affine deformation and orientation of optically anisotropic fibrils constituting the films and orientation toward the stretching direction of the principal optical axes of the scattering elements constituting the fibrils.

The effect of birefringence on the light-scattering patterns was also studied for the case when the stretching direction of the films was inclined with respect to the polarization direction of the polarizer or analyzer used in the polarized light-scattering method.

\section{KEY WORDS Light-Scattering / Films / Fibrils / Model / Rod /} Calculation / Affine Deformation / Birefringence /
\end{abstract}

The light-scattering method has been used to characterize crystalline superstructures of polymer films. ${ }^{1-7}$ The scattering patterns which are typical and have been most frequently found in the crystalline polymer films are those from the spherulitic crystalline superstructure. ${ }^{2,3,7,8}$ The origin of the scattering has been shown to be essentially described in terms of optically anisotropic spheres ${ }^{2,3}$ or disks ${ }^{7,8}$ for the undeformed spherulites, and ellipsoids ${ }^{9,10}$ or ellipses ${ }^{7}$ for the deformed spherulites.

The light-scattering patterns of the type which are quite different from the spherulitic scatter-

* Presented partially at the Annual Meeting of the Society of Polymer Science, Japan, Tokyo, May 24, 1972.

** Present Address: The Central Research Laboratories, Mitsubishi Chemical Industries, Inc., Hisamoto-Kamoi-cho, Kawasaki, Japan.

*** Present Address: Research Laboratories, Niihama Factory, Sumitomo Chemical Co. Ltd., Niihama, Ehime, Japan.

**** To whom all correspondence should be addressed. ing and which are designated as "rod-like scattering" have been occasionally found for polymeric films such as poly(tetrafluoroethylene), ${ }^{6,11,12}$ poly(chlorotrifluoroethylene), ${ }^{13}$ native cellulose,${ }^{14}$ collagen, ${ }^{4,15}$ amylose ${ }^{5}$ etc. The origin of the rod-like scattering has been shown to arise from a nonspherulitic or prespherulitic structure which is composed of more or less randomly-spaced optically-anisotropic fibrils. The fibrils observed under a light or electron microscope are thought to be a crystal lammella or a stack of crystal lammellae in case of poly (tetrafluoroethylene) and poly(chlorofluoroethylene). ${ }^{16}$

The scattering from the fibrillar structure was first described by Stein, et al., in terms of one dimensional anisotropic rods distributed randomly in a two-dimensional plane normal to propagation direction of the incident beam. ${ }^{12}$ Further extensions and refinements of the model calculation have been worked out for cases of one dimensional rods in three-dimensional space $^{4,27}$ and three-dimensional rods in two ${ }^{17}$ 
or three-dimensional space. ${ }^{17-19}$

The results of such calculations have shown that the important features of the experimental scattering patterns can be described, at least qualitatively, in terms of the model based upon the random assembly of isolated, homogeneous and anisotropic rods.

Changes in the scattering patterns with deformation of films having a fibrillar structure were studied by Stein, et al., ${ }^{12}$ Samuels, ${ }^{20}$ and Chien, et al. ${ }^{15}$ and were explained in terms of (i) orientation of the rods and (ii) orientation of principal optical axis direction within the rods. These quantities were empirically introduced into the calculations.

In this report, the effects of stretching on $H_{\mathrm{V}}$ and $V_{\mathrm{V}}$ light-scattering patterns were studied for several films having the fibrillar structure. The effects which were commonly seen for these samples at an initial stage of the deformation were explained in terms of a modified model in which the optical axes of the scattering elements within the rods orient affinely toward the stretching direction, and the rods themselves are deformed and oriented according to the affine deformation.

The calculations were carried out for two cases, one in which the stretching direction was set parallel to the polarization direction of the polarizer, i.e., the case where the effect of birefringence on the light scattering is minimized, and the other in which the stretching direction was set inclined to the polarization direction of the polarizer, i.e., the case in which the effect of birefringence should be significant, as will be shown later.

\section{TEST SPECIMENS AND EXPERIMENTAL SCATTERING PATTERNS}

The scattering patterns for stretched films were taken under $H_{\mathrm{V}}$ polarization where the polarization directions of the polarizer and analyzer are set in vertical and in horizontal directions, respectively, and under $V_{\mathrm{V}}$ polarization where the polarization directions of the polarizer and analyzer are set in vertical directions. The stretching direction of the films was set parallel to the vertical direction so that as will be discussed later, the effect of birefringence on the scattering patterns is minimized except for the case where the effect of birefringence was studied as in Figure 6.

In Figure 1, the change of $H_{\mathrm{V}}$ light-scattering patterns upon stretching a collagen film is shown. The collagen films were cast from solubilized collagen by proteolipic enzymes except collagenase, which were designated as RC-I in the previous paper. ${ }^{4}$ The films were first immersed in $0.9-\%$ saline solution and then stretched by a stretching device. The stretched films were then pressed lightly by plates made out of acryl resin so as to flatten the film surfaces. The films were washed thoroughly by distilled water and subsequently dried in air.

In Figure 2 the effect of stretching on the $H_{\mathrm{V}}$ patterns of (polychlorotrifluoroethylene) films is shown. The polymer powder was melted at $270^{\circ} \mathrm{C}$ and pressed under laboratory hot plates for $10 \mathrm{~min}$ and subsequently quenched in a water bath at room temperature. The films thus made were put in a silicon oil bath held at $217^{\circ} \mathrm{C}$ for $10 \mathrm{~min}$, and then rapidly transferred into an another oil bath whose temperature was held at $144^{\circ} \mathrm{C}$ for $5 \mathrm{~min}$.

Figures 3 and 4 show the effect of stretching upon $H_{\mathrm{V}}$ and $V_{\mathrm{V}}$ patterns of the hydroxypropylcellulose films, respectively, which were cited in a paper by Samuels. ${ }^{20}$

In these figures, the $H_{\mathrm{V}}$ patterns for all the unstretched films show a four-fold symmetrical intensity distribution with maximum intensities at azimuthal angle $(\mu) 0^{\circ}$ (in meridian) and $90^{\circ}$ (in equator). The intensity continuously decreases with increasing $\theta$, the scattering angle. Consequently the patterns are typical of those from a random assembly of anisotropic fibrils with their principal optical axes inclined by an angle of about $45^{\circ}$ with respect to the fibril axes.

$V_{\mathrm{V}}$ scattering depends upon the density fluctuation as well as the orientation fluctuation, while $H_{\mathrm{V}}$ scattering depends only upon the orientation fluctuation., ${ }^{1,2}$ The $V_{\mathrm{V}}$ patterns from the unstretched films except for the hydroxypropylcellulose films were circularly symmetrical around the incident beam axis, indicating that the scattering predominantly arises from the density fluctuations for these films, while the scattering 
N. Hayashi, Y. Murakami, M. Moritani, T. Hashimoto, and H. Kawai

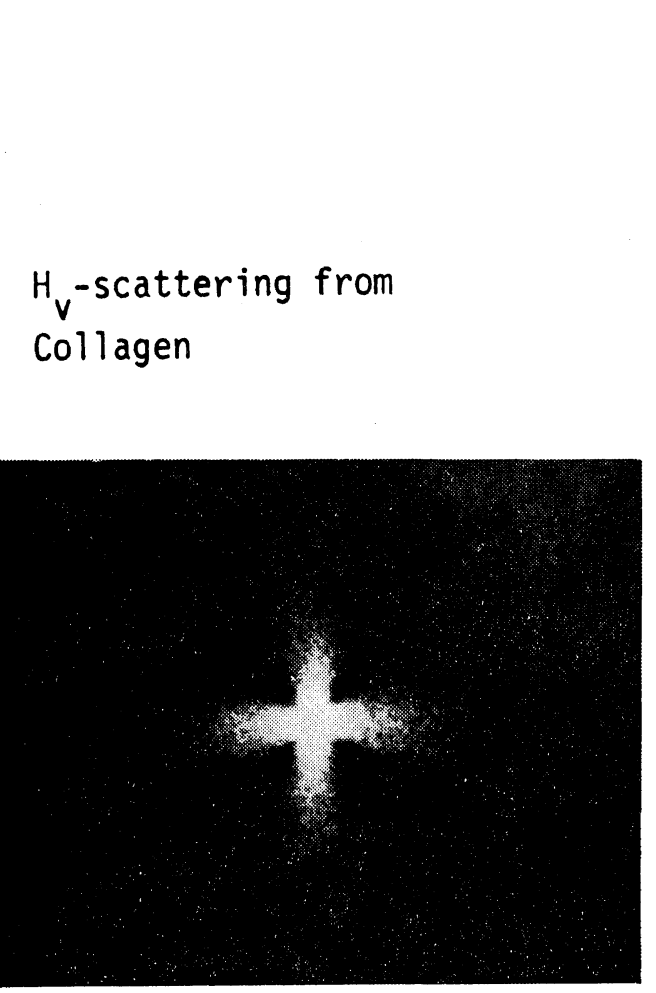

(a) Unstretched

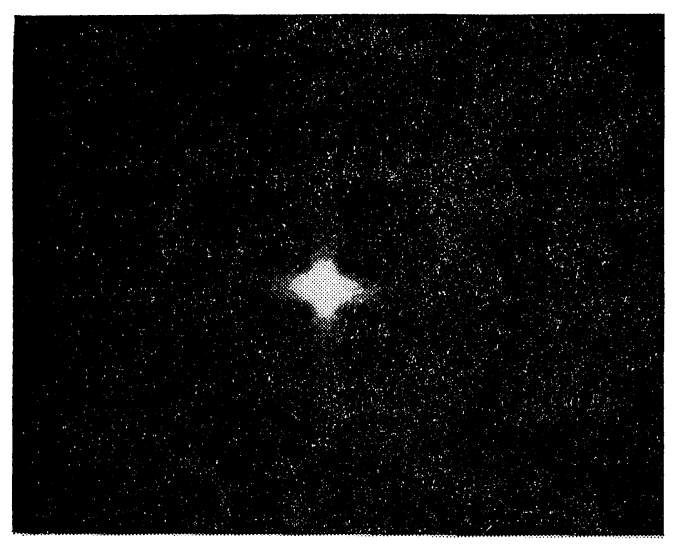

(b) $25-\%$ Stretched

\section{Stre.Direc.}
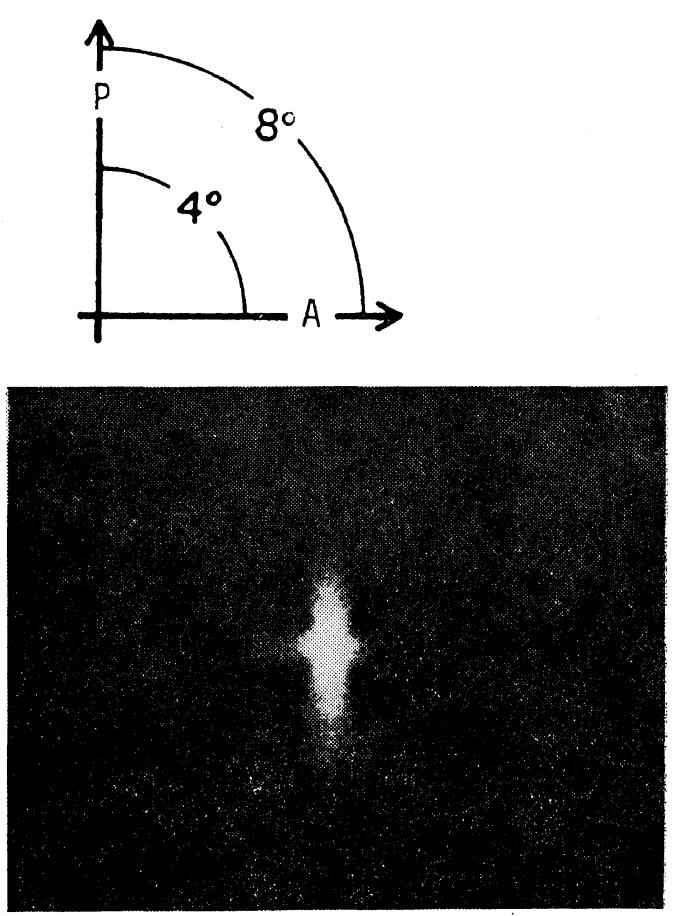

(c) $40-\%$ Stretched

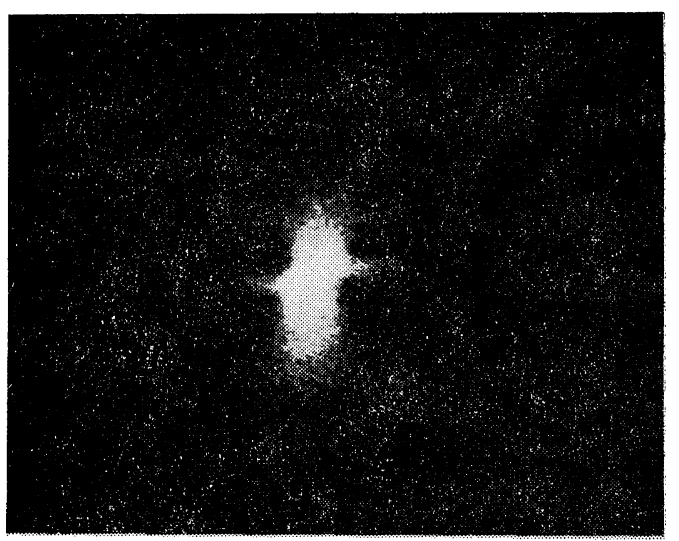

(d) $60-\%$ Stretched

Figure 1. Change of $H_{\mathrm{V}}$ scattering patterns upon stretching the collagen films by (a) $0 \%$, (b) $25 \%$, (c) $40 \%$, and (d) $60 \%$. The stretching direction is vertical.

from the films of hydroxypropylcellulose is anisotropic, indicating that the contribution of the orientation fluctuation predominates.

A generalization which may be made from examination of the changes of the $H_{\mathrm{V}}$ patterns upon stretching these films is that the meridional lobes of the patterns (i.e., the lobes at $\mu=$ 0 and $180^{\circ}$ ) become diffuse and thick, while 

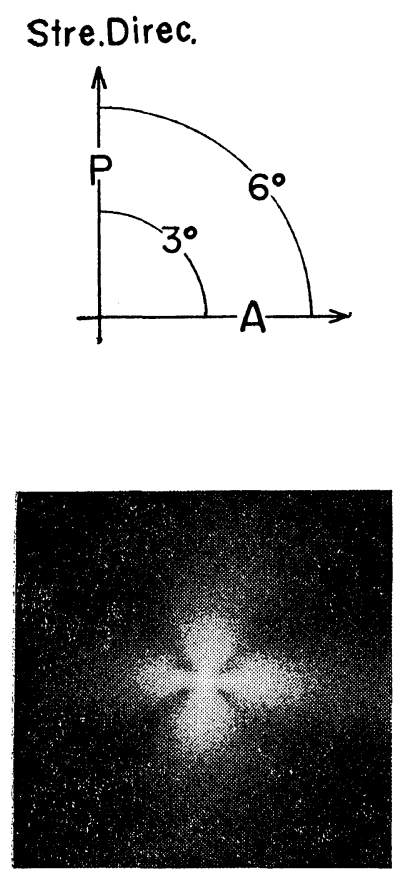

(c) 30\%-Stretched

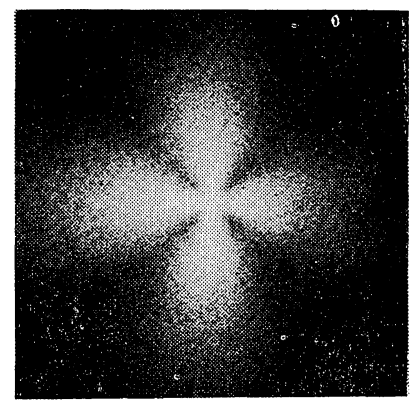

(a) Unstrefched

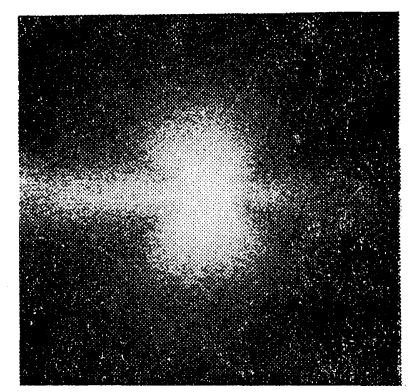

(d) $53 \%$-Stretched

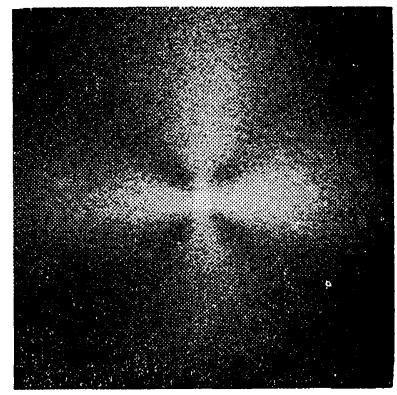

(b) $10 \%$-Stretched

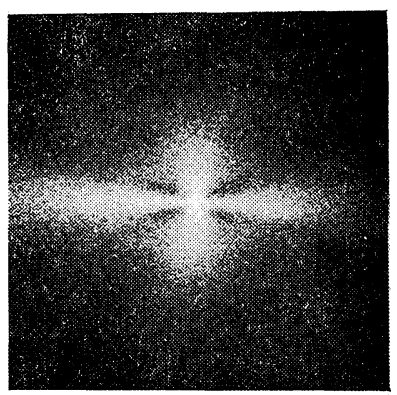

(e) $85 \%$-Stretched

Figure 2. Change of $H_{\mathrm{V}}$ scattering patterns upon stretching the poly(chlorotrifluoroethylene) films by (a) $0 \%$, (b) $10 \%$, (c) $30 \%$, (d) $53 \%$, and (e) $85 \%$. The stretching direction is vertical.

the equatorial lobes (i.e., the lobes at $\mu=90$ and $270^{\circ}$ ) become sharp and thin. A similar trend was observed by Rhodes and Stein ${ }^{12}$ upon stretching poly(tetrafluoroethylene) films. The origin of the characteristic change of the patterns observed in the initial stage of elongation will be discussed later. It should be noted, however, that the patterns at a given elongation ratio are quantitatively different for the different polymer films. This indicates that local strain imposed on the fibrils and the deformability of the fibrils at a given strain are different for the different films.

The changes of the $V_{V}$ patterns upon stretching are more complicated than those of the $H_{\mathrm{V}}$ patterns because of the density contribution. In the case of the collagen films, the initially circular symmetrical pattern for the unstretched film (Figure 5b) seems to become an anisotropic pattern upon stretching by $60 \%$ (Figure $5 d$ ), which results from a change in the relative contribution of the density and orientation fluctuations upon stretching. On the other hand, for the hydroxypropylcellulose films where the orientation contribution is significant, the lobes with their maximum intensities at odd multiples of $\mu=45^{\circ}$ in the unstretched films orient toward the equator in the initial elongation as shown in Figure 4.

A similar stretching effect upon the $H_{\mathrm{V}}$ patterns is also seen for polypropylene and polyethylene A-B type block copolymer films as shown in Figure $6 .^{21}$ The block copolymer was prepared by the Ziegler-Natta method. The intrinsic viscosity of the copolymer was 1.08 , and the mole fraction of the ethylene component was $20 \%$. The copolymer powder was preheated at $185^{\circ} \mathrm{C}$ for $15 \mathrm{~min}$, and pressed under a laboratory press at $185^{\circ} \mathrm{C}$ for $15 \mathrm{~min}$, and subsequently quenched in water at $20^{\circ} \mathrm{C}$. The films thus made were annealed at $125^{\circ} \mathrm{C}$ for $30 \mathrm{~min}$.

The $H_{\mathrm{V}}$ scattering patterns for the films are complicated and are composed of two types 
N. Hayashi, Y. Murakami, M. Moritani, T. Hashimoto, and H. Kawai $\mathrm{H}_{\mathrm{V}}$-scattering from

Hydroxypropylcellulose
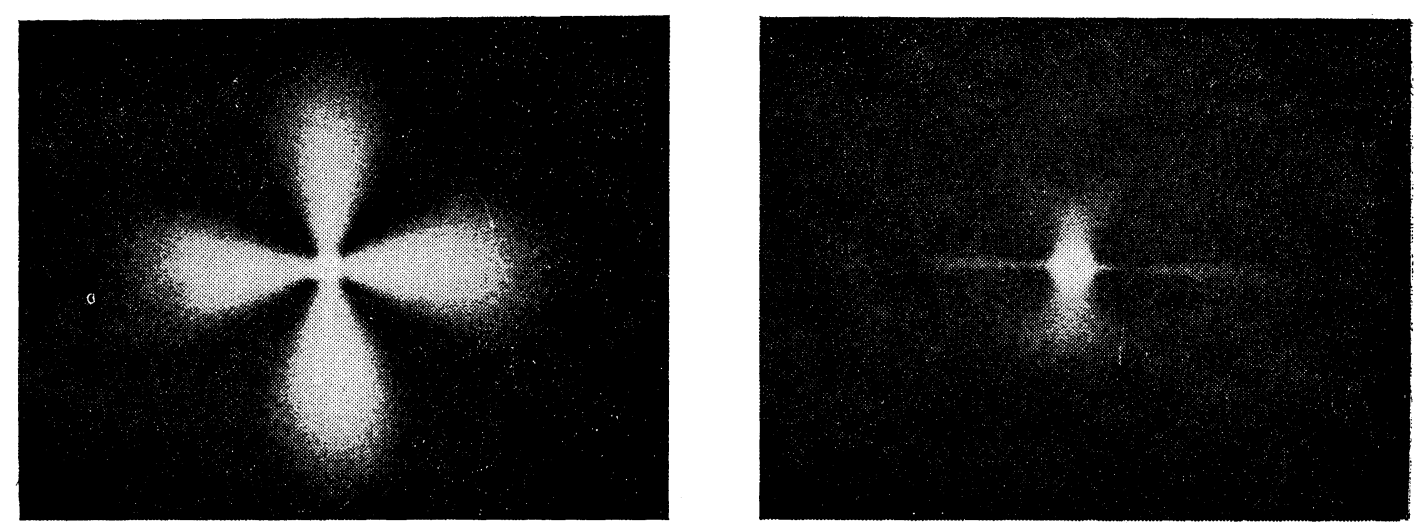

(a) Unstretched

(d) $150-\%$ Stretched
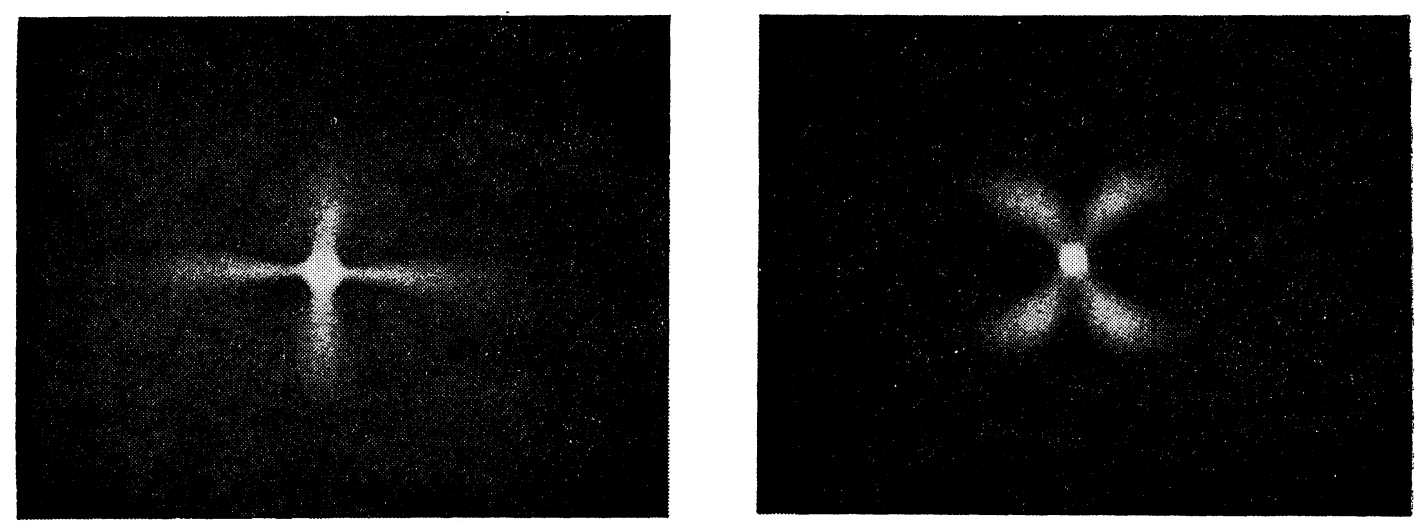

(b) $50-\%$ stretched

(e) $200-\%$ Stretched
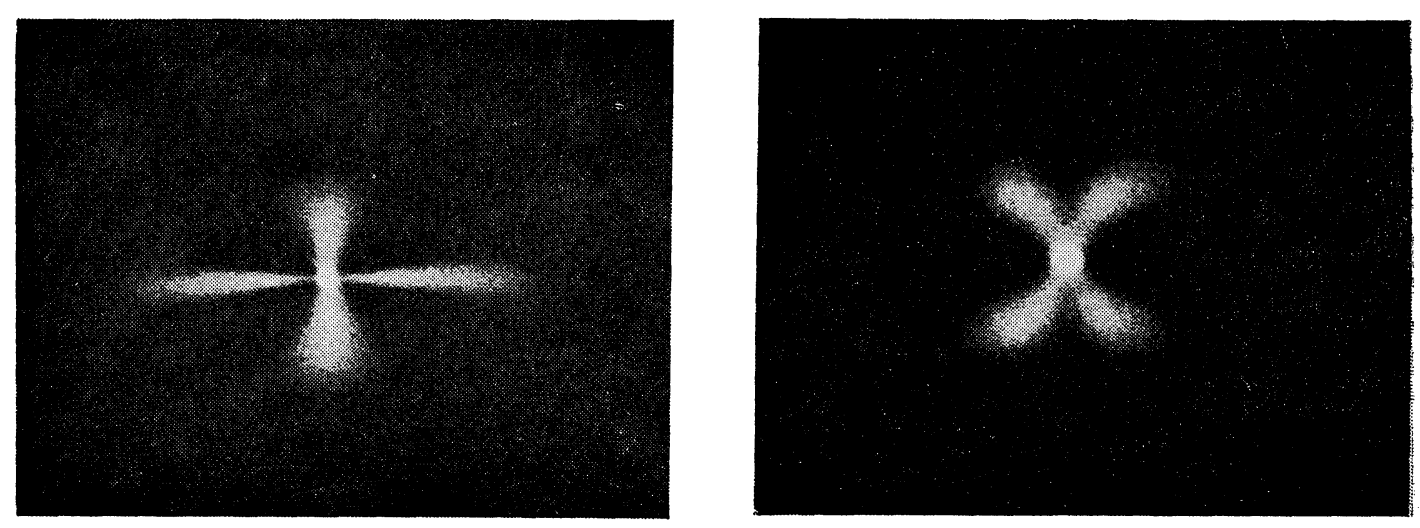

(c) $100-\%$ stretched

(f) $250-\%$ Stretched

Figure 3. Change of $H_{\mathrm{V}}$ scattering patterns upon stretching the water soluble hydroxypropylcellulose films by (a) $0 \%$, (b) $50 \%$, (c) $100 \%$, (d) $150 \%$, (e) $200 \%$, and (f) $250 \%$. The stretching direction is vertical. (Data by R. J. Samuels ${ }^{19}$.) 
$v_{v}$-scattering from

Hydroxypropylcel1ulose

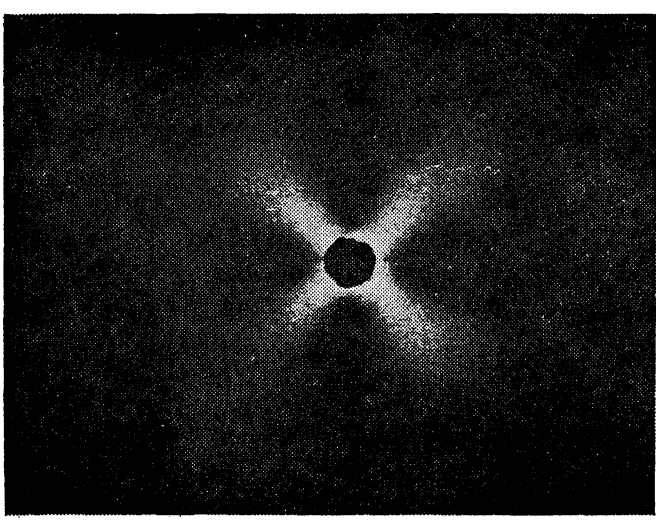

(a) Unstretched

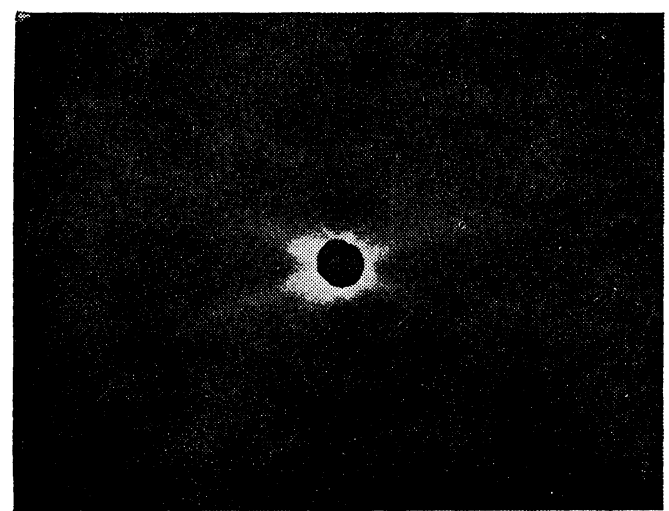

(b) $50-\%$ Stretched

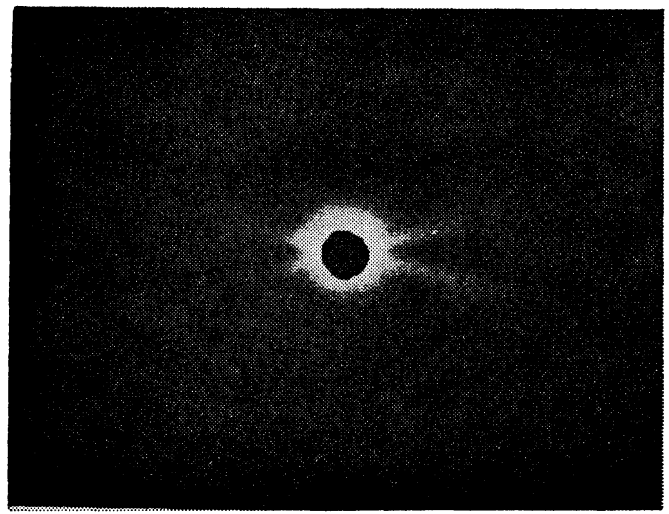

(c) $100-\%$ Stretched

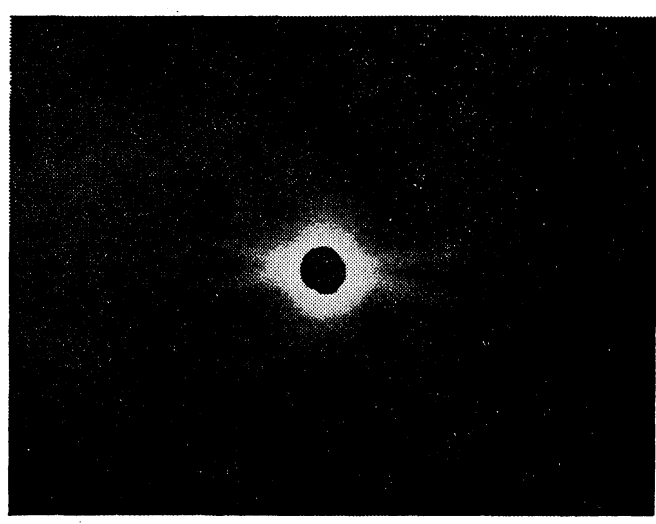

(d) $150-\%$ stretched

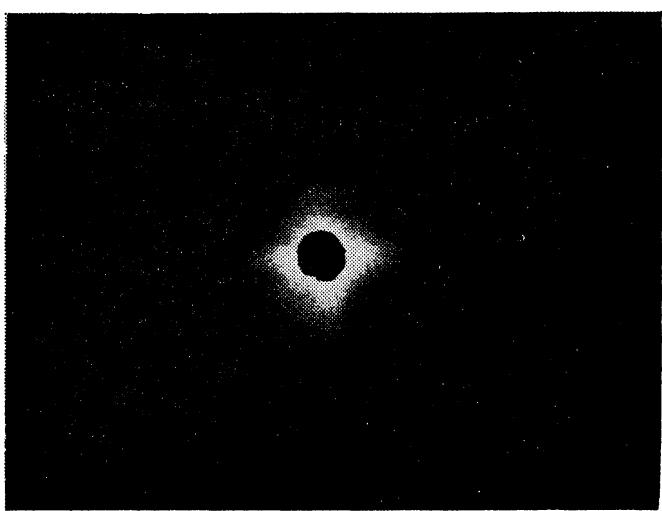

(e) $200-\%$ Stretched

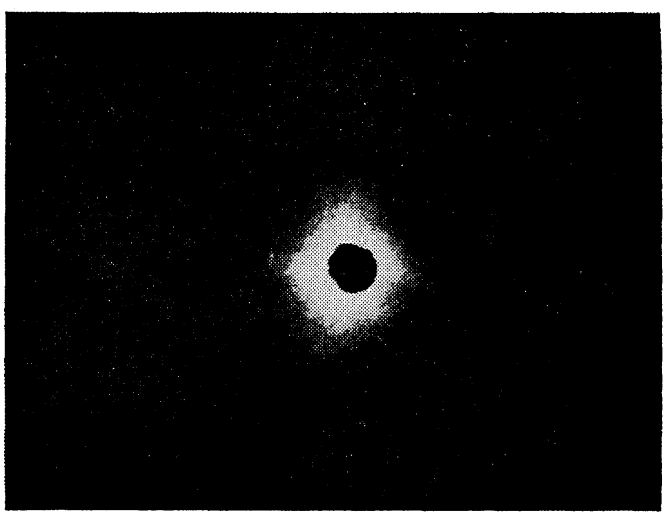

(f) $250-\%$ Stretched

Figure 4. Change of $V_{\mathrm{V}}$ patterns upon stretching the water soluble hydroxypropylcellulose films by (a) $0 \%$, (b) $50 \%$, (c) $100 \%$, (d) $150 \%$, (e) $200 \%$ and (f) $250 \%$. The stretching direction is vertical. (Data by R. J. Samuels. ${ }^{19}$ ) 


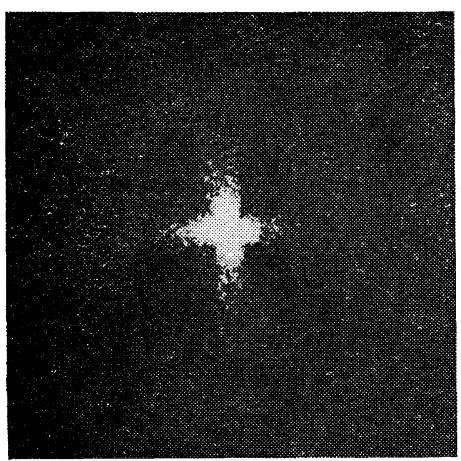

(a) $H_{v}$, unstretched

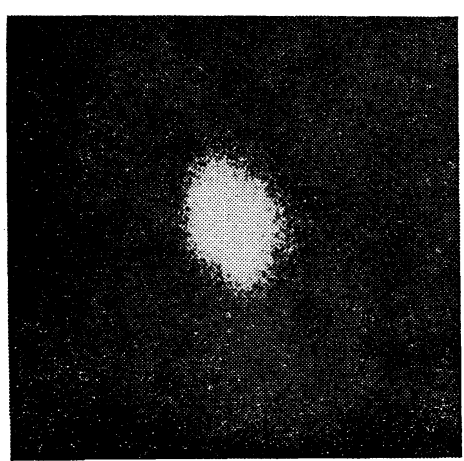

(e) $H_{v}, 60-\%$ stretche, $X=-15^{\circ}$

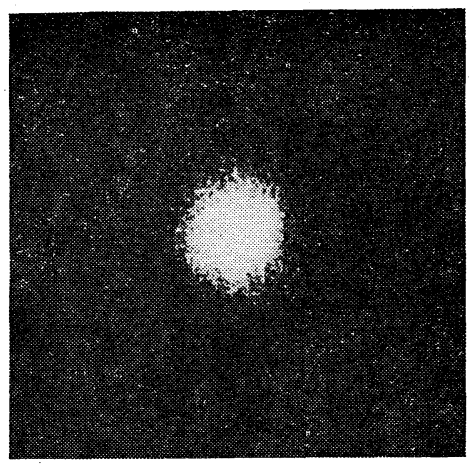

(b) $v_{v}$, unstretched

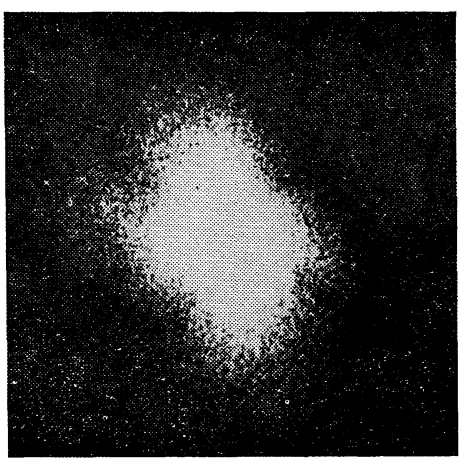

(f) $\mathrm{H}_{\mathrm{v}}, 60-\%$ stretched, $X=-30^{\circ}$

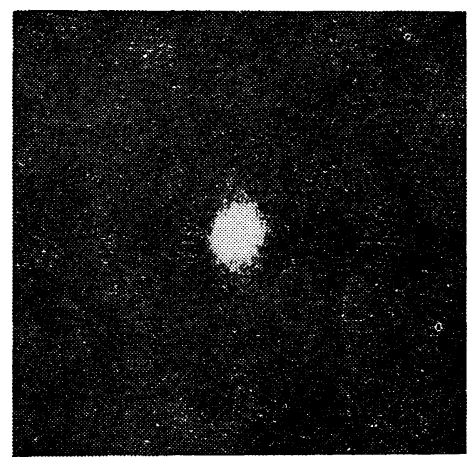

(c) $\mathrm{H}_{\mathrm{v}}, 60-\%$ stretched, $x=0^{\circ}$

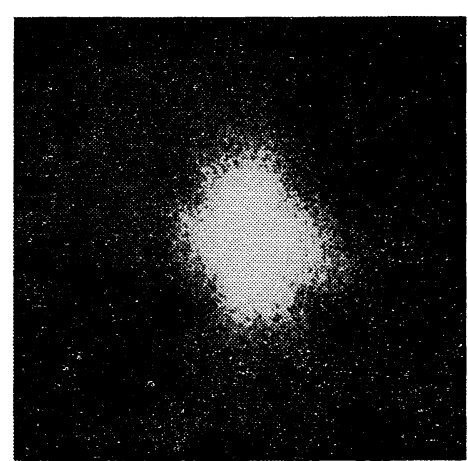
(g) $\begin{aligned} H_{v} & =60-\% \text { stretched, } \\ X & =-45^{\circ}\end{aligned}$

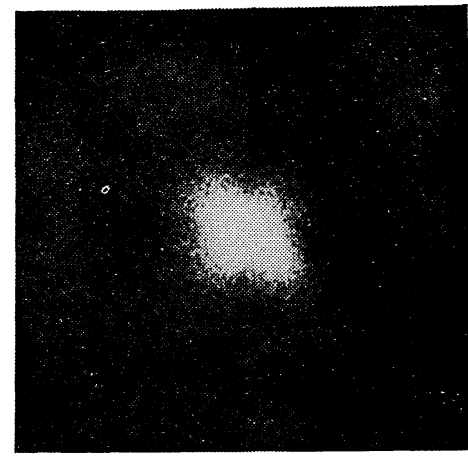

(d) $v_{v}, 60-\%$ stretched, $\chi=0^{0}$

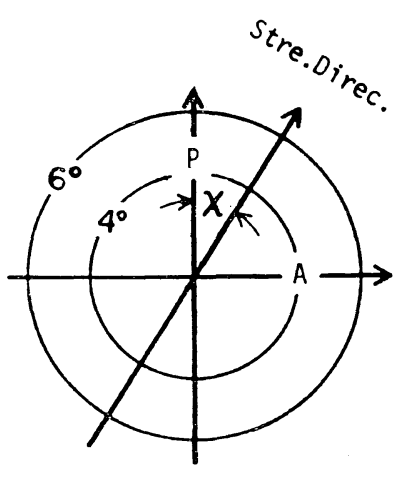

2

Figure 5. Effect of stretching on $H_{\mathrm{V}}$ and $V_{\mathrm{V}}$ fatterns of the collagen films under the condition in which the stretching directicn is inclined by an angle $\chi$ from the vertical direction. (a) and (b) are the $H_{\mathrm{V}}$ and $V_{\mathrm{V}}$ pattenns for undeformed films, respectively, and (c) and (d) are the $H_{\mathrm{V}}$ and $V_{\mathrm{V}}$ patterns for stretched films by $60 \%$ under $\chi=0^{\circ}$, respectively. (e), (f), and (g) are the $H_{\mathrm{V}}$ patterns for stretched films by $60 \%$ under $\chi=-15,-30$, and $45^{\circ}$, respectively. 
Light-Scattering Patterns from Stretched Films

$\mathrm{H}_{\mathrm{V}}$-scattering from

80/20 P-E Block Copolymer

Stre.Direc.

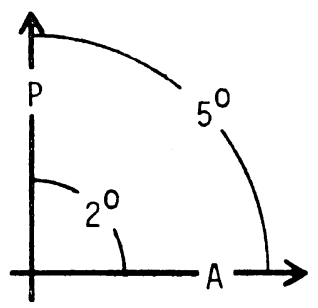

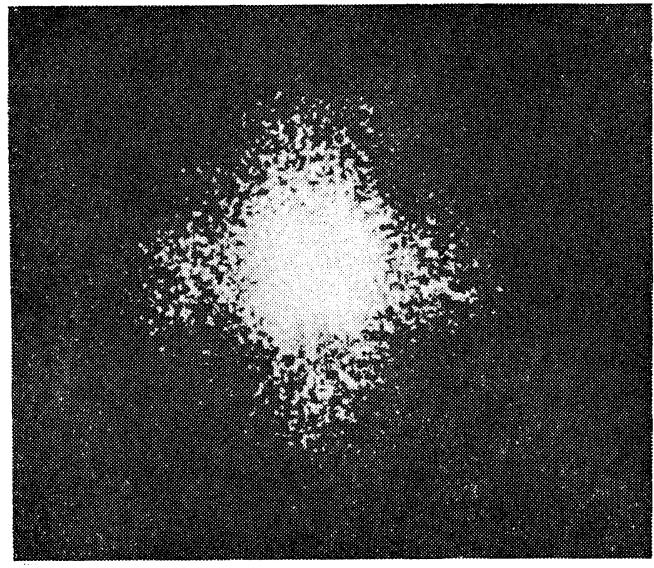

Unstretched

(a)

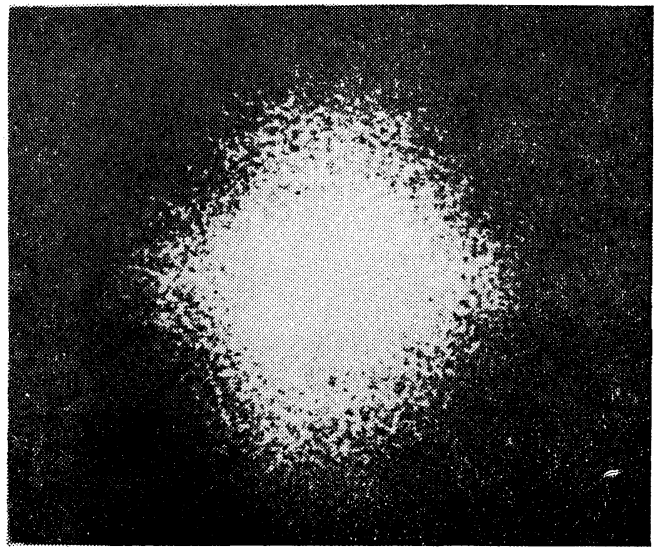

$10-\%$ stretched

(b)

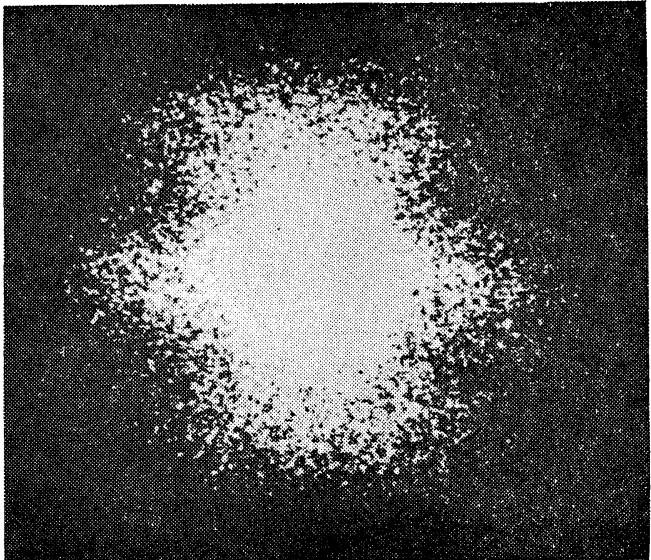

40-\% stretched

(c)

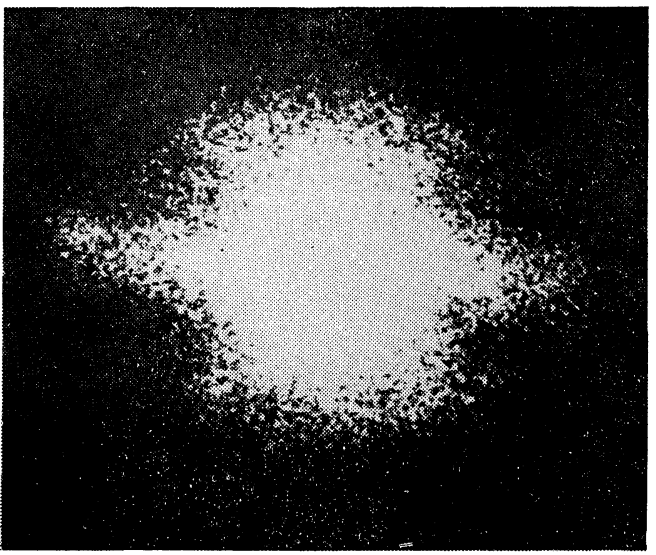

78-\% stretched

(d)

Figure 6. Change of $H_{\mathrm{V}}$ patterns upon stretching the polyethylene, polypropylene A-B type block copolymer films by (a) $0 \%$, (b) $10 \%$, (c) $40 \%$, and (d) $78 \%$. The stretching direction is vertical. 

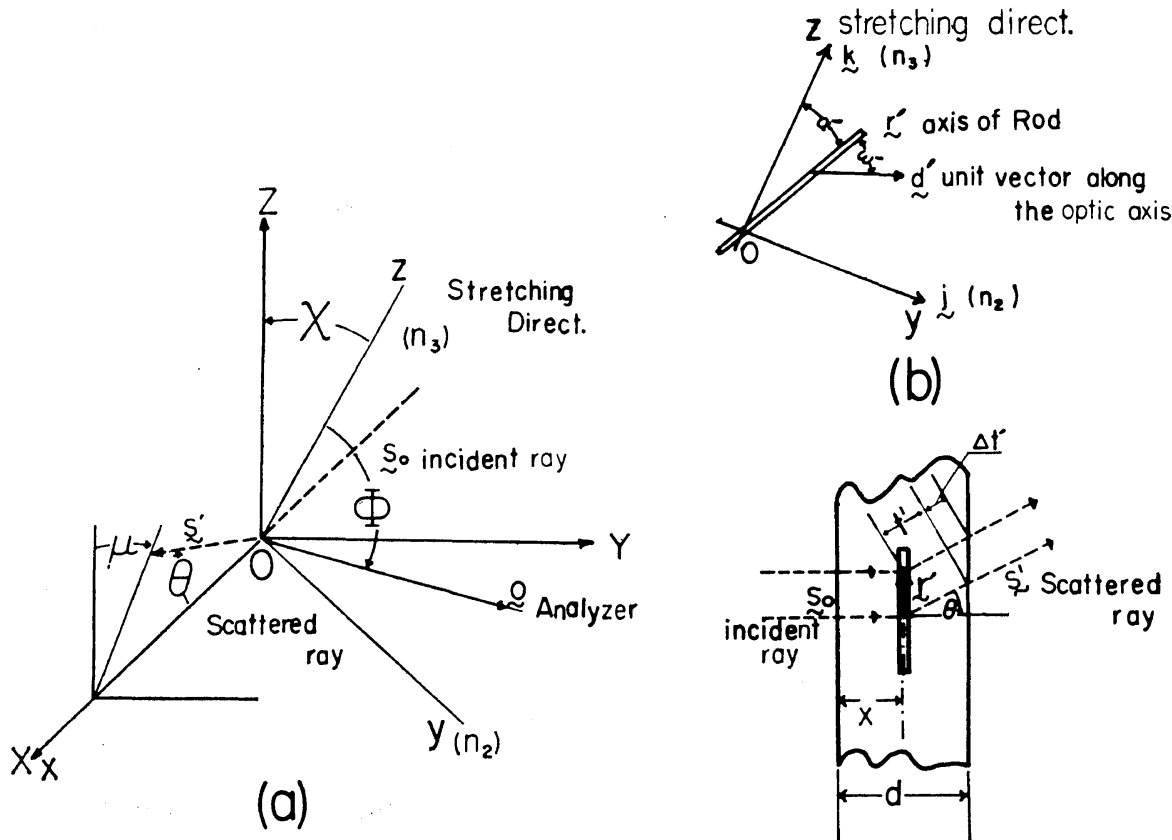

(b)

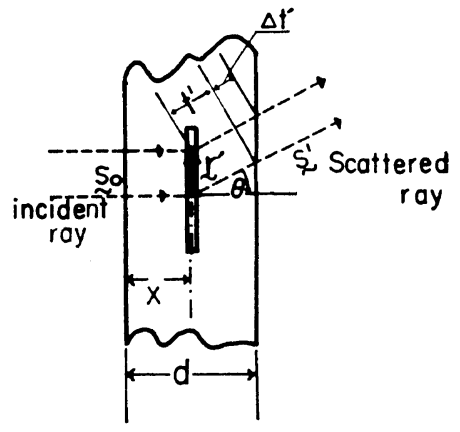

(c)

Figure 7. Definitions of the coordinate systems and variables used in the calculations.

of patterns as shown in Figure 6: (i) the spherulitic scattering at very small scattering angle with a four-leaf clover appearance and (ii) the rod-like scattering at large scattering angles with lobes extended in the meridional and equatorial directions. The origin of the coexistence of the two types of patterns will not be discussed here. It should be noted, however, that the rod-like scattering changes very similarly to those demonstrated previously for other films having typical fibrillar structure.

In Figure 5, the effect of birefringence on the rod-like scattering from the collagen films is shown. The retardation of the films was about $\pi / 5 \mathrm{rad}$. The $H_{\mathrm{V}}$ (a) and $V_{\mathrm{V}}$ (b) scattering from the unstretched films, and the $H_{\mathrm{V}}$ (c) and $V_{\mathrm{V}}$ (d) scattering from the stretched films with the stretching direction parallel to the polarization direction of the polarizer are typical of those from the unoriented and oriented rod-like assemblies, respectively. When the stretching direction is rotated counterclockwise by an angle $\chi$ from the vertical direction (see Figure 7), the $H_{\mathrm{V}}$ and $V_{\mathrm{V}}$ scattering patterns are seen to be diffused and distorted. The distortion seems to be a maximum at $\chi= \pm 45^{\circ}$ in which the incident and scattered beams undergo maximum retardation within the stretched specimen. Consequently the distortion seems to arise partially from the effect of birefringence on the scattering, which will be discussed later.

\section{THEORETICAL CALCULATIONS}

In order to understand the characteristic change of $H_{\mathrm{V}}$ and $V_{\mathrm{V}}$ patterns upon the initial elongation of the films as discussed before, the calculations were carried out using several models. Among the models employed only that which turned out to be most effective in describing the characteristic change of the patterns will be discussed.

In Figure $7 \mathrm{a}$, coordinate systems and angles used in the calculations are defined. $O-X Y Z$ coordinate is the one fixed to the apparatus where $O Z$ - and $O X$ - axes are set parallel to the 
vertical direction of the apparatus and to the propagation direction of the incident beam whose unit vector is defined as $\mathbf{s}_{0}$, respectively. $i_{1}, j_{1}$, and $k_{1}$ are the unit vectors along the $O X, O Y$-, and $0 Z$ - axes, respectively. The scattered beam from the films whose propagation direction is defined as $s^{\prime}$ is observed as a function of $\theta$ and $\mu$ through an analyzer whose polarization direction is defined as a unit vector $\mathbf{O}$ as shown in the figure. The stretching direction along the unit vector $\mathbf{k}$ is set at an angle $\chi$ from $0 Z$ - axis in the $0 Y Z$ - plane. At small scattering angles, $\mathbf{O}$, which is set perpendicular to $s^{\prime}$, is approximated to lie in a plane parallel to the plane of $0 Y Z$. The $0-x y z$ coordinate is the one fixed to the sample where the $0 z$ axis is the one along the stretching direction, and the rod-like structure is assumed to be in the plane of $0 y z$ perpendicular to the $0 X$ - axis. In Figure $7 b, \boldsymbol{r}^{\prime}$ is a vector along the rod axis and the angle $\alpha^{\prime}$ is the angle which $\boldsymbol{r}^{\prime}$ makes with respect to $k$, the unit vector along the stretching direction. $\mathbf{d}^{\prime}$ is a unit vector along the principal optical axis of the scattering element within the rod which makes an angle $\omega^{\prime}$ with respect to $\boldsymbol{r}^{\prime}$.

The calculations were carried out for two cases: one case in which the angle $\chi$ is set to be zero, so that the effect of birefringence is minimized and the other case in which the angle $\chi$ is set arbitrarily, so that the effect of birefringence should be taken into account. The former case will be considered first and then the latter case.

\section{Calculation under the Minimum Birefringence Effect}

The case where $\chi$ is zero, i.e., the case where the stretching direction is set parallel to the vertical direction will be considered and the calculations restricted to the case in which the rods are homogeneous and identical, and interference of the scattered waves from the different rods negligible. The rods are assumed to be in the plane $O Y Z$ and to be infinitely thin in lateral dimensions.

Although the treatment based upon the restrictions loses its quantitative applicability to analyses of the experimental patterns, it has been shown to be still useful in order to describe qualitatively fundamental importances of the experimental scattering patterns from previous work..$^{4,5,12,15,17-20}$

The effect of the finite lateral dimension of the rods, ${ }^{17-19}$ inhomogeneities with the rods, ${ }^{22}$ size distribution of the rods ${ }^{22}$ and the manner of spatial distribution ${ }^{17}$ (two-dimensional or three-dimensional distribution) on the scattering patterns were studied separately for unoriented systems. The results show that the effects do affect quantitatively the scattering intensity distributions of the patterns but that they do not alter qualitatively important appearances of the patterns. Hence the significance of the treatment based upon the restrictions is still maintained in that sense.

The amplitude of scattered light from the anisotropic rod with a given orientation $\alpha$ with respect to $0 z$-axis in unstretched state is given by

$$
E=C \int_{-L / 2}^{L / 2} \rho(\boldsymbol{M} \cdot \mathbf{O}) \cos [k(\boldsymbol{r} \cdot s)] \mathrm{d} r
$$

$C$ is a constant related to the absolute intensity of scattering, and $\rho$ is the scattering power per unit length of the rod. $L$ is the length of the rods and $k$ is defined as $2 \pi / \lambda^{\prime}$ where $\lambda^{\prime}$ is the wavelength of light in the medium.

$M$ is the induced dipolemoment of the scattering element located at a distance $r$ from the center of the rod, and is given for vertical polarization of the polarizer by

$$
\boldsymbol{M}=E_{0}\left[\delta\left(\mathbf{k}_{1} \cdot \mathbf{d}\right) \mathbf{d}+b_{\mathrm{t}} \boldsymbol{k}_{1}\right]
$$

$E_{0}$ is the amplitude of the incident beam. The scattering element is assumed to have uniaxial anisotropy with polarizabilities $\alpha_{\| /}$and $\alpha_{\perp}$ along and perpendicular to the optical axis, respectively. $d$ is the unit vector along the optical axis in the unstretched state. If the polarizability in the surrounding medium of the rods is $\alpha_{\mathrm{s}}$, then

$$
\delta=b_{\mathrm{r}}-b_{\mathrm{t}}, \quad b_{\mathrm{r}}=\alpha_{/ /}-\alpha_{\mathrm{s}}, \quad \text { and } \quad b_{\mathrm{t}}=\alpha_{\perp}-\alpha_{\mathrm{s}}
$$

The unit vector $\mathbf{O}$ along the polarization direction of the analyzer for the horizontal polarization, is given by,

$$
\mathbf{O}=\mathbf{O}_{\mathrm{H}}=-\sin \rho_{2} \mathbf{i}_{1}+\cos \rho_{2} \mathbf{j}_{1}
$$

and for vertical polarization, 


$$
\mathbf{O}=\mathbf{O}_{\mathrm{v}}=-\sin \rho_{1} \mathbf{i}_{1}+\cos \rho_{1} \mathbf{k}_{1}
$$

where $\rho_{1}$ and $\rho_{2}$ were previously evaluated. ${ }^{7,23}$ At very small $\theta, \mathbf{O}_{\mathrm{H}}$ and $\mathbf{O}_{\mathrm{\nabla}}$ reduce to

$$
\mathbf{O}_{\mathrm{H}}=\mathbf{j}_{1}, \quad \mathbf{O}_{\mathrm{V}}=\mathbf{k}_{1}
$$

$\boldsymbol{r}$ and $\boldsymbol{s}$ are the vectors along the rod axis in the unstretched state and the scattering vector defined as $\mathbf{s}_{0}-\boldsymbol{s}^{\prime}$, respectively. The scattered intensity from the assembly, on the basis of the assumption of independent scattering, is then given by

$$
I=\int_{0}^{2 \pi} N(\alpha)|E|^{2} \mathrm{~d} \alpha
$$

$N(\alpha)$ is a normalized distribution function for the orientation of the rod axes which is constant $\left(N_{0}\right)$ in unstretched films. Since the rods are homogeneous, $(\boldsymbol{M} \cdot \mathbf{O})$ in eq 1 is independent of $r$, so that it can be put outside the integral.

When the films are stretched along the $0 z$ axis, the rods themselves may be deformed and oriented toward the stretching direction, and, in addition, the optical axes of the scattering elements within the rods may be oriented toward the stretching direction, so that the equations should be modified as follows,

$$
\begin{gathered}
E^{\prime}=C \int_{-L^{\prime} / 2}^{L^{\prime} / 2} \rho^{\prime}\left(\boldsymbol{M}^{\prime} \cdot \mathbf{O}\right) \exp \left[i k\left(\boldsymbol{r}^{\prime} \cdot \boldsymbol{s}\right)\right] \mathrm{d} \boldsymbol{r}^{\prime} \\
\boldsymbol{M}^{\prime}=E_{0}\left[\delta^{\prime}\left(\mathbf{k}_{1} \cdot \mathbf{d}^{\prime}\right) \mathbf{d}^{\prime}+b_{\mathrm{t}}{ }^{\prime} \boldsymbol{k}_{1}\right]
\end{gathered}
$$

and

$$
I^{\prime}=\int_{0}^{2 \pi} N^{\prime}\left(\alpha^{\prime}\right)\left|E^{\prime}\right|^{2} \mathrm{~d} \alpha^{\prime}
$$

where the primed quantities are those corresponding to the stretched state.

The scattered intensity $I^{\prime}$ is then calculated for a certain model. The model which was employed is based upon the assumptions that (a) the rods are deformed and oriented toward the stretching direction according to affine deformation process and that (b) the optical axes of the scattering elements within the rods are also oriented toward the stretching direction according to the affine deformation.

In Figure 8, the model is schematically shown. The rod of length $L$, initially oriented at a given angle $\alpha$ with respect to the $0 z$ axis, is deformed into the rod of length $L^{\prime}$ and oriented

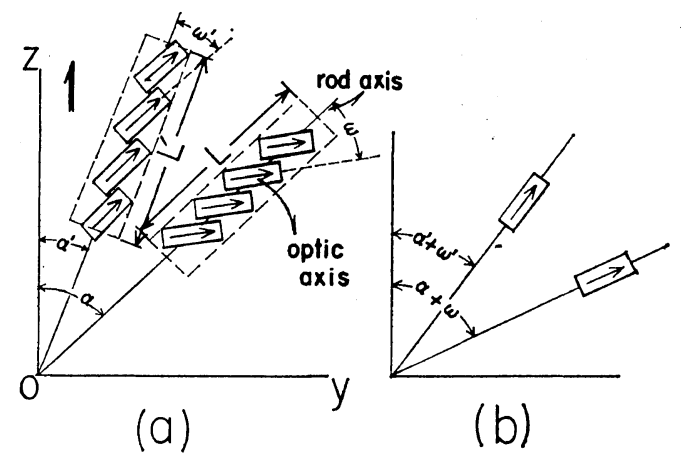

Figure 8. The model of affine deformation of the anisotropic rods.

at angle $\alpha^{\prime}$ (Figure 8a). Accompanied by the deformation process, the optical axes of the scattering elements change their orientation from $(\alpha+\omega)$ to $\left(\alpha^{\prime}+\omega^{\prime}\right)$ with respect to the stretching direction (Figure $8 b$ ). Such deformation processes might be realized when the fibrils are composed of the scattering elements interconnected by a weak physical force. The change of the optical axis orientation is in part associated with the deformation processes of sliding, rotation, bowing and kinking of lamellae observed by Speerschneider and $\mathrm{Li}^{24}$ on stretching poly(tetrafluoroethylene) films.

The affine deformation of the rod leads to

$$
\begin{aligned}
\boldsymbol{r}^{\prime} & =r^{\prime}\left[\sin \alpha^{\prime} \mathbf{j}+\cos \alpha^{\prime} \mathbf{k}\right] \\
& =r\left[\lambda_{2} \sin \alpha \mathbf{j}+\lambda_{3} \cos \alpha \mathbf{k}\right]
\end{aligned}
$$

where $\lambda_{3}$ and $\lambda_{2}$ are elongation ratios imposed on the rod along and perpendicular to the stretching direction. The eq 8 implies that the deformation of the rods with respect to their length is angularly dependent. Similarly the orientation of the rods is given, according to the affine deformation, by

$$
N^{\prime}\left(\alpha^{\prime}\right) \mathrm{d} \alpha^{\prime}=N_{0} \mathrm{~d} \alpha
$$

or

$$
\begin{aligned}
\frac{N^{\prime}\left(\alpha^{\prime}\right)}{N_{0}} & =\frac{\mathrm{d} \alpha}{\mathrm{d} \alpha^{\prime}}=\left(\lambda_{3}{ }^{2} \cos ^{2} \alpha+\lambda_{2}{ }^{2} \sin ^{2} \alpha\right) / \lambda_{2} \lambda_{3} \\
& =\lambda_{2} \lambda_{3}\left(\lambda_{2}{ }^{2} \cos ^{2} \alpha^{\prime}+\lambda_{3}{ }^{2} \sin ^{2} \alpha^{\prime}\right)^{-1}
\end{aligned}
$$

where $N_{0}$ is the orientation distribution function of the rods for the unstretched films which is independent of $\alpha$. The optical axis orientation in the stretched state is also given, according 
to the affine deformation, by

$$
\begin{aligned}
d^{\prime} & =\sin \left(\alpha^{\prime}+\omega^{\prime}\right) \mathbf{j}+\cos \left(\alpha^{\prime}+\omega^{\prime}\right) \mathbf{k} \\
& =\frac{\lambda_{2} \sin (\alpha+\omega) \mathbf{j}+\lambda_{3} \cos (\alpha+\omega) \mathbf{k}}{\left[\lambda_{2}{ }^{2} \sin ^{2}(\alpha+\omega)+\lambda_{3}{ }^{2} \cos ^{2}(\alpha+\omega)\right]^{1 / 2}}
\end{aligned}
$$

The total scattering power of the rod may be unchanged on stretching, so that

$$
\rho \mathrm{d} r=\rho^{\prime} \mathrm{d} r^{\prime}
$$

Now from eq 3 and $5-11, H_{\mathrm{V}}$ and $V_{\mathrm{V}}$ scattering intensities $\left(I_{H_{V}}\right.$ and $I_{V_{V}}$, respectively) from the system may be given by

$$
\begin{gathered}
I_{H_{\mathrm{V}}}=K^{\prime} \int_{0}^{2 \pi}\left(\boldsymbol{M}^{\prime} \cdot \mathbf{O}\right)^{2}{ }_{H_{\mathrm{V}}} H^{2} \mathrm{~d} \alpha \\
I_{V_{\mathrm{V}}}=K^{\prime} \int_{0}^{2 \pi}\left(\boldsymbol{M}^{\prime} \cdot \mathbf{O}\right)^{2}{ }_{V_{\mathrm{V}}} H^{2} \mathrm{~d} \alpha
\end{gathered}
$$

where

$$
\left(\boldsymbol{M}^{\prime} \cdot \mathbf{O}\right)_{H_{\mathrm{V}}}=E_{0} \delta^{\prime} \frac{\cos (\alpha+\omega) \sin (\alpha+\omega)}{\lambda_{2}^{2} \sin ^{2}(\alpha+\omega)+\lambda_{3}{ }^{2} \cos ^{2}(\alpha+\omega)}
$$

$$
\begin{aligned}
& \left(\boldsymbol{M}^{\prime} \mathbf{O}\right)_{V_{\mathrm{V}}} \\
& =E_{0}\left[\delta^{\prime} \frac{\lambda_{3}{ }^{2} \cos ^{2}(\alpha+\omega)}{\lambda_{2}{ }^{2} \sin ^{2}(\alpha+\omega)+\lambda_{3}{ }^{2} \cos ^{2}(\alpha+\omega)}+b_{\mathrm{t}}{ }^{\prime}\right]
\end{aligned}
$$

$$
H=\frac{\sin \left[U\left(\lambda_{2} \sin \mu \sin \alpha+\lambda_{3} \cos \mu \cos \alpha\right)\right]}{U\left(\lambda_{2} \sin \mu \sin \alpha+\lambda_{3} \cos \mu \cos \alpha\right)}
$$

and

$$
U=(\pi L \sin \theta) / \lambda^{\prime}
$$

Equations $12-17$ are derived for a given value of $\omega$, the angle that the optic axis makes with respect to the rod axis in the unstretched state. Since positive and negative values of $\omega$ are equally probable, the equations should be averaged for $+\omega$ and $-\omega$.

Effect of Birefringence on the Rod-Like Scattering

Now the calculations will be extended to the more general case in which the stretching direction is inclined by an angle $\chi$ from the polarization direction of the polarizer which again is set parallel to the vertical direction. Under this condition, the effect of retardation which the incident and scattered beams undergo when they traverse the birefringent specimen should be taken into account, as mentioned earlier.

The principles of the calculations are essential- ly the same as that used in the previous works on the effect of birefringence on spherulitic scattering. ${ }^{25,26}$

In Figure $7 \mathrm{c}$, a schematic diagram of the optical paths of the incident and scattered beams is shown in which the diagram is seen from the $0 Y$-axis in Figure 7a. First we will calculate $E_{3}$, the amplitude of the scattering from a scattering element at a distance $r^{\prime}$ from the center of the rod located at a position $x$ in the stretched specimen and oriented with an angle $\alpha^{\prime}$ with respect to the stretching direction (Figures $7 \mathrm{~b}$ and $7 \mathrm{c}$ ). Then the scattered amplitude from the rod, $E_{x}\left(\alpha^{\prime}\right)$ is given by

$$
E_{x}\left(\alpha^{\prime}\right)=\int_{-L^{\prime} / 2}^{L^{\prime} / 2} \rho^{\prime}\left(\boldsymbol{E}_{3} \cdot \mathbf{O}\right) \mathrm{d} r^{\prime}
$$

where $E_{3}$ includes the phase factor as will be shown in eq 23 and 24 . The scattered intensity $I_{\mathrm{b}}$ is given by

$$
I_{\mathrm{b}}=\int_{x=0}^{d} \int_{\alpha^{\prime}=0}^{2 \pi} N^{\prime}\left(\alpha^{\prime}\right) E_{x}\left(\alpha^{\prime}\right) E_{x}{ }^{*}\left(\alpha^{\prime}\right) \mathrm{d} \alpha^{\prime} \mathrm{d} x
$$

$\boldsymbol{E}_{3}$ in eq 18 which corresponds to $C M^{\prime} \exp \left[i k\left(\boldsymbol{r}^{\prime}\right.\right.$. $s)$ ] in eq 5 is subjected to the retardation.

The effect of retardation on $E_{3}$ is evaluated as follows. Vertically polarized incident field strength $\boldsymbol{E}_{0}$ is retarded to result in $\boldsymbol{E}_{1}$ when it traverses the specimen and arrives at the scattering element at the position $x$ in the specimen. The incident field $\boldsymbol{E}_{1}$ is then scattered by the scattering element. The scattered amplitude $\boldsymbol{E}_{2}$ is again retarded to result is $\boldsymbol{E}_{3}$ when the scattered beam traverses the distance $t^{\prime}+\Delta t^{\prime}$ in the specimen and leaves the surface.

If $\boldsymbol{E}_{0}$ is defined as

$$
\boldsymbol{E}_{0}=\left[E_{0} \exp (i \omega t)\right] \mathbf{k}_{1}
$$

then $E_{1}$ is given by

$$
E_{1}=\left(\boldsymbol{E}_{0} \cdot \mathbf{k}\right) \mathbf{k} \exp \left(i k_{0} n_{3} x\right)+\left(\boldsymbol{E}_{0} \cdot \mathbf{j}\right) \mathbf{j} \exp \left(i k_{0} n_{2} x\right)
$$

where $n_{3}$ and $n_{2}$ are refractive indicies of the specimen along and perpendicular to the stretching direction, respectively (Figure 7b). $k_{0}$ is defined as $2 \pi / \lambda_{0}$ where $\lambda_{0}$ is wavelength of light in vacuo. The scattered amplitude $\boldsymbol{E}_{2}$ from the scattering element is given, in a manner similar to obtaining eq 6 , by

$$
E_{2}=C\left[\delta^{\prime}\left(E_{1} \cdot \mathbf{d}^{\prime}\right) \mathbf{d}^{\prime}+b_{\mathrm{t}}^{\prime} E_{1}\right]
$$


Now at a small scattering angle $\theta, \Delta t^{\prime}$ is negligibly small compared with $t^{\prime}$, and a vector perpendicular to $s^{\prime}$ in the plane of $s_{0}$ and $s^{\prime}$ is almost parallel to $\mathbf{k}$, a unit vector along the stretching direction. Consequently $\boldsymbol{E}_{3}$ can be approximated with good accuracy by

$$
\begin{aligned}
\boldsymbol{E}_{3}= & \left(\boldsymbol{E}_{2} \cdot \mathbf{k}\right) \mathbf{k} \exp \left(i k_{0} n_{3} t^{\prime}\right) \\
& +\left[\boldsymbol{E}_{2}-\left(\boldsymbol{E}_{2} \cdot \mathbf{k}\right) \mathbf{k}\right] \exp \left(i k_{0} n_{2} t^{\prime}\right)
\end{aligned}
$$

and from Figure $7 \mathrm{c}$ it follows that

$$
t^{\prime}=[(d-x) / \cos \theta]-\left(\boldsymbol{r}^{\prime} \cdot \mathbf{s}^{\prime}\right)
$$

where $d$ is thickness of the specimen.

Now from Figures $7 a$ and $7 b$ it follows that

$$
\begin{gathered}
\mathbf{O}=\sin \Phi \mathbf{j}+\cos \Phi \mathbf{k} \quad(\text { at small } \theta) \\
\boldsymbol{r}^{\prime}=r^{\prime}\left[\cos \alpha^{\prime} \mathbf{k}+\sin \alpha^{\prime} \mathbf{j}\right]
\end{gathered}
$$

$\boldsymbol{s}^{\prime}=\cos \theta \mathbf{i}_{1}+\sin \theta \sin (\mu-\chi) \mathbf{j}_{1}+\sin \theta \cos (\mu-\chi) \mathbf{k}_{1}$

$$
\begin{gathered}
\left(\boldsymbol{E}_{0} \cdot \mathbf{k}\right)=E_{0} \exp (i \omega t) \cos \chi \\
\left(\boldsymbol{E}_{0} \cdot \mathbf{j}\right)=E_{0} \exp (i \omega t) \sin \chi \\
\mathbf{d}^{\prime}=\sin \left(\alpha^{\prime}+\omega^{\prime}\right) \mathbf{j}+\cos \left(\alpha^{\prime}+\omega^{\prime}\right) \mathbf{k}
\end{gathered}
$$

By combining the eq 18 , and $20-29$ and using the eq 11, it follows that

$$
\begin{aligned}
& E_{x}\left(\alpha^{\prime}\right) E_{x}{ }^{*}\left(\alpha^{\prime}\right)=C^{2} \rho^{2}\left\{\operatorname { c o s } ^ { 2 } \Phi \left[R_{1}{ }^{2}+R_{2}{ }^{2}\right.\right. \\
& \left.\quad-2 R_{1} R_{2} \cos \left(k_{0} \Delta_{\mathrm{s}} t^{\prime}\right)\right] M_{1}+R_{2}{ }^{2} M_{2} \\
& \left.\quad+2 \cos \Phi R_{1} R_{2}\left(M_{3}-M_{4}\right)\right\}
\end{aligned}
$$

where

$$
\begin{gathered}
R_{1}=L \sin Z / Z, \quad R_{2}=L \sin Z^{\prime} / Z^{\prime} \\
Z=\left(\pi L / \lambda_{0}\right) n_{3} \sin \theta \cos \left(\alpha^{\prime}-\mu+\chi\right) \\
Z^{\prime}=\left(\pi L / \lambda_{0}\right) n_{2} \sin \theta \cos \left(\alpha^{\prime}-\mu+\chi\right)
\end{gathered}
$$

and $\Delta_{\mathrm{s}}$ is the birefringence of the specimen defined as $\left(n_{3}-n_{2}\right)$. The quantities $M_{1}, M_{2}, M_{3}$, and $M_{4}$ are given as follows,

$$
\begin{aligned}
M_{1}= & \left(\delta^{\prime}\right)^{2} \cos ^{2}\left(\alpha^{\prime}+\omega^{\prime}\right) \\
& \times\left[\cos ^{2} \chi \cos ^{2}\left(\alpha^{\prime}+\omega^{\prime}\right)+\sin ^{2} \chi \sin ^{2}\left(\alpha^{\prime}+\omega^{\prime}\right)\right. \\
& \left.+\frac{1}{2} \sin 2 \chi \sin 2\left(\alpha^{\prime}+\omega^{\prime}\right) \cos \left(k_{0} \Delta_{\mathrm{s}} x\right)\right] \\
& +\left(b_{\mathrm{t}}{ }^{\prime}\right)^{2} \cos ^{2} \chi+2 \delta^{\prime} b_{\mathrm{t}}{ }^{\prime} \cos \left(\alpha^{\prime}+\omega^{\prime}\right) \\
& \times\left[\cos ^{2} \chi \cos \left(\alpha^{\prime}+\omega^{\prime}\right)\right. \\
& \left.+\frac{1}{2} \sin 2 \chi \sin \left(\alpha^{\prime}+\omega^{\prime}\right) \cos \left(k_{0} \Delta_{\mathrm{s}} x\right)\right] \\
M_{2}= & \left(\delta^{\prime}\right)^{2} \cos ^{2}\left(\alpha^{\prime}+\omega^{\prime}-\Phi\right) \\
& \times\left[\cos ^{2} \chi \cos ^{2}\left(\alpha^{\prime}+\omega^{\prime}\right)+\sin ^{2} \chi \sin ^{2}\left(\alpha^{\prime}+\omega^{\prime}\right)\right.
\end{aligned}
$$

$\left.+\frac{1}{2} \sin 2 \chi \sin 2\left(\alpha^{\prime}+\omega^{\prime}\right) \cos \left(k_{0} \Delta_{\mathrm{s}} x\right)\right]$

$+\left(b_{t}{ }^{\prime}\right)^{2}\left[\cos ^{2} \Phi \cos ^{2} \chi+\sin ^{2} \Phi \sin ^{2} \chi\right.$

$\left.+\frac{1}{2} \sin 2 \Phi \sin 2 \chi \cos \left(k_{0} \Delta_{\mathrm{s}} x\right)\right]$

$+2 \delta^{\prime} b_{\mathrm{t}}{ }^{\prime} \cos \left(\alpha^{\prime}+\omega^{\prime}-\Phi\right)$

$\times\left[\cos \Phi \cos ^{2} \chi \cos \left(\alpha^{\prime}+\omega^{\prime}\right)\right.$

$+\sin \Phi \sin ^{2} \chi \sin \left(\alpha^{\prime}+\omega^{\prime}\right)$

$\left.+\frac{1}{2} \sin 2 \chi \sin \left(\Phi+\alpha^{\prime}+\omega^{\prime}\right) \cos \left(k_{0} \Delta_{\mathrm{s}} x\right)\right]$

$M_{3}=\left(\delta^{\prime}\right)^{2} \cos \left(\alpha^{\prime}+\omega^{\prime}\right) \cos \left(\alpha^{\prime}+\omega^{\prime}-\Phi\right)$

$\times\left[\cos ^{2} \chi \cos ^{2}\left(\alpha^{\prime}+\omega^{\prime}\right)+\sin ^{2} \chi \sin ^{2}\left(\alpha^{\prime}+\omega^{\prime}\right)\right.$

$\left.+\frac{1}{2} \sin 2 \chi \sin 2\left(\alpha^{\prime}+\omega^{\prime}\right) \cos \left(k_{0} \Delta_{\mathrm{s}} x\right)\right]$

$\times \cos \left(k_{0} \Delta_{\mathrm{s}} t^{\prime}\right)+\left(b_{\mathrm{t}}{ }^{\prime}\right)^{2}$

$\times\left[\cos \Phi \cos ^{2} \chi \cos \left(k_{0} \Delta_{\mathrm{s}} t^{\prime}\right)\right.$

$+\frac{1}{2} \sin \Phi \sin 2 \chi \cos \left[k_{0} \Delta_{\mathrm{s}}\left(x+t^{\prime}\right)\right]$

$+\left(\delta^{\prime} b_{\mathrm{t}}\right)^{2}\left[2 \cos \Phi \cos ^{2} \chi \cos ^{2}\left(\alpha^{\prime}+\omega^{\prime}\right)\right.$

$\left.+\frac{1}{2} \sin \Phi \sin 2\left(\alpha^{\prime}+\omega^{\prime}\right)\right] \cos \left(k_{0} \Delta_{\mathrm{s}} t^{\prime}\right)$

$+\frac{1}{4} \cos \Phi \sin 2 \chi \sin 2\left(\alpha^{\prime}+\omega^{\prime}\right)$

$\times \cos \left[k_{0} \Delta_{\mathrm{s}}\left(x-t^{\prime}\right)\right]+\frac{1}{2}[\sin \Phi \sin 2 \chi$

$\left.+\frac{1}{2} \cos \Phi \sin 2 \chi \sin 2\left(\alpha^{\prime}+\omega^{\prime}\right)\right]$

$\times \cos \left[k_{0} \Delta_{\mathrm{s}}\left(x+t^{\prime}\right)\right]$

$M_{4}=\left(\delta^{\prime}\right)^{2} \cos \left(\alpha^{\prime}+\omega^{\prime}\right) \cos \left(\alpha^{\prime}+\omega^{\prime}-\Phi\right)$

$\times\left[\cos ^{2} \chi \cos ^{2}\left(\alpha^{\prime}+\omega^{\prime}\right)+\sin ^{2} \chi \sin ^{2}\left(\alpha^{\prime}+\omega^{\prime}\right)\right.$

$\left.+\frac{1}{2} \sin 2 \chi \sin 2\left(\alpha^{\prime}+\omega^{\prime}\right) \cos \left(k_{0} \Delta_{\mathrm{s}} x\right)\right]+\left(b_{\mathrm{t}}{ }^{\prime}\right)^{2}$

$\times\left[\cos \Phi \cos ^{2} \chi+\frac{1}{2} \sin \Phi \sin 2 \chi \cos \left(k_{0} \Delta_{\mathrm{s}} x\right)\right]$

$+\left(\delta^{\prime}\right)\left(b_{\mathrm{t}}{ }^{\prime}\right)\left[2 \cos \Phi \cos ^{2} \chi \cos ^{2}\left(\alpha^{\prime}+\omega^{\prime}\right)\right.$

$+\frac{1}{2} \sin \Phi \sin 2\left(\alpha^{\prime}+\omega^{\prime}\right)$

$+\frac{1}{2} \sin \Phi \sin 2 \chi \cos \left(k_{0} \Delta_{\mathrm{s}} x\right)$

$\left.+\frac{1}{2} \cos \Phi \sin 2 \chi \sin 2\left(\alpha^{\prime}+\omega^{\prime}\right) \cos \left(k_{0} \Delta_{\mathrm{s}} x\right)\right]$

In the case when the specimen is unstretched and not birefringent, $\Delta_{\mathrm{s}}$ is equal to zero, which leads to the coefficient of $M_{1}$ being zero, and $\left(M_{3}-M_{4}\right)$ being zero. Consequently only the $M_{2}$ term contributes to the scattering. Noticing that $\alpha^{\prime}$ and $\omega^{\prime}$ reduce to $\alpha$ and $\omega$, respectively, and that $N^{\prime}\left(\alpha^{\prime}\right)$ is equal to $N_{0}$, for $H_{\nabla}$ scattering in which $\mathbf{k}_{1}=\mathbf{k}$, i.e., $\chi=0$, and $\Phi$ is approximately $90^{\circ}$ at small $\theta$, it follows that

$$
\begin{aligned}
& E_{x}\left(\alpha^{\prime}\right) E_{x}{ }^{*}\left(\alpha^{\prime}\right)=C^{2} L^{2} \delta^{2} \sin ^{2}(\alpha+\omega) \cos ^{2}(\alpha+\omega) \\
& \quad \times \frac{\sin ^{2}\left[\frac{1}{2} k_{0} \operatorname{Ln} \sin \theta \cos (\alpha-\mu)\right]}{\left[\frac{1}{2} k_{0} \operatorname{Ln} \sin \theta \cos (\alpha-\mu)\right]^{2}}
\end{aligned}
$$

where $n$ is the refractive index of the specimen. Consequently the equation reduces to the previ- 
ous equation for $H_{\mathrm{V}}$ scattering from the unoriented systems. Similarly for $V_{\mathrm{V}}$ scattering $\chi=\Phi=0^{\circ}$, and the equation for $E_{x}\left(\alpha^{\prime}\right) E_{x}{ }^{*}\left(\alpha^{\prime}\right)$ reduces to the previous case.

Previously considered $H_{\mathrm{V}}$ and $V_{\mathrm{V}}$ scatterings under $\chi=0^{\circ}$ from the stretched films can be also derived from eq 30 . In case of $H_{\mathrm{V}}$ scattering, $\chi=0^{\circ}$ and $\Phi=90^{\circ}$ so that the coefficient of $M_{2}$ is equal to zero, and $\left(M_{3}-M_{4}\right)$ is also zero.

Consequently

$$
E_{x}\left(\alpha^{\prime}\right) E_{x}{ }^{*}\left(\alpha^{\prime}\right)=C^{2} L^{2} R_{2}{ }^{2} M_{2}
$$

where

$$
M_{2}=\left(\delta^{\prime}\right)^{2} \sin ^{2}\left(\alpha^{\prime}+\omega^{\prime}\right) \cos ^{2}\left(\alpha^{\prime}+\omega^{\prime}\right)
$$

and $R_{2}$ is given by eq 31 and 33 .

In eq 39, the birefringence effect on the $H_{\mathrm{V}}$ scattering appears through the quantity $n_{2}$ in the term of $R_{2} . \quad n_{2}$ is given in terms of refractive index $n$ and birefringence $\Delta_{\mathrm{s}}$ of the specimen as

$$
n_{2}=n-\left(\Delta_{s} / 3\right)
$$

In normal experimental situations, $n \gg \Delta_{\mathrm{s}} / 3$ so that $n_{2} \cong n$.

Consequently the effect of birefringence on the $H_{\mathrm{V}}$ light scattering is negligible, and in this condition, the result of eq 39 is identical to the previous result obtained from eq 5,6 , and 11.

Similarly $V_{\mathrm{V}}$ scattering is obtained from eq 30 as

$$
\begin{aligned}
E_{x}\left(\alpha^{\prime}\right) E_{x}{ }^{*}\left(\alpha^{\prime}\right)= & C^{2} L^{2}\left[\delta^{\prime} \cos ^{2}\left(\alpha^{\prime}+\omega^{\prime}\right)\right. \\
& \left.+b_{\mathrm{t}}\right]^{2}\left(R_{1}{ }^{2}+2 R_{2}{ }^{2}-2 R_{1} R_{2}\right)
\end{aligned}
$$

Since $n \gg \Delta_{\mathrm{s}}, R_{1} \cong R_{2}$, so that the effect of birefringence is also negligibly small for the $V_{\mathrm{V}}$ scattering too, and eq 42 reduces to the previous equation for the $V_{\mathrm{V}}$ scattering.

The integration over $x$ in eq 19 can now be easily carried out and is composed of the following terms,

$$
\begin{gathered}
D_{1}=\int_{0}^{d} \cos \left(k_{0} \Delta_{\mathrm{s}} x\right) \mathrm{d} x=(\mathrm{d} \sin \Gamma) / \Gamma \\
D_{2}=\int_{0}^{d} \cos \left[k_{0} \Delta_{\mathrm{s}}(d-x) / \cos \theta\right] \mathrm{d} x \\
=(d \cos \theta / \Gamma) \sin (\Gamma / \cos \theta) \\
D_{3}=\int_{0}^{d} \cos \left[k_{0} \Delta_{\mathrm{s}}\left(x+\frac{d-x}{\cos \theta}\right)\right] \mathrm{d} x
\end{gathered}
$$

Polymer J., Vol. 4, No. 5, 1973

$$
=d[\sin \Gamma-\sin (\Gamma / \cos \theta)] /[\Gamma-(\Gamma / \cos \theta)]
$$

and

$$
\begin{aligned}
D_{4} & =\int_{0}^{d} \cos \left[k_{0} \Delta_{\mathrm{s}}\left(x-\frac{d-x}{\cos \theta}\right)\right] \mathrm{d} x \\
& =d[\sin \Gamma+\sin (\Gamma / \cos \theta)] /[\Gamma+\Gamma / \cos \theta]
\end{aligned}
$$

where $\Gamma=k_{0} \Delta_{\mathrm{s}} d$, the retardation of the specimen.

The calculation of the integration over $\alpha^{\prime}$ in eq 19 can be made for a given model of the oriented rods. The previous model was used for the calculation.

\section{RESULTS OF THE CALCULATIONS AND COMPARISON WITH THE EXPERIMENTAL PATTERNS}

Numerical calculations of the results were carried using the FACOM 230-60 Computer at the Research Computer Center of Kyoto University.

In order to facilitate comparisons with the experimental patterns, further approximations were introduced to reduce the number of parameters; (i) the local elongation ratios $\lambda_{2}$ and $\lambda_{3}$ are given by $\lambda_{1}{ }^{-1}$ and $\lambda_{1}$, respectively, based on the assumption of deformation under constant volume, and (ii) $\delta^{\prime}$ and $b_{\mathrm{t}}{ }^{\prime}$ are independent of $\alpha^{\prime}$, the angular position of the rod. From the second approximation $\delta^{\prime}$ and $b_{t}{ }^{\prime}$ become constants which implicitly depend upon $\lambda_{1}$ and which can be put outside the integrals in eq 12, 13, and 19. Thus, in case of $H_{\nabla}$ scattering under $\chi=0^{\circ}$, it is seen from eq 12 and 14 that change of $\delta^{\prime}$ upon stretching affects only the absolute intensity but not the relative intensity distribution. On the other hand, from eq 13 and 15 , the intensity distribution of $V_{\nabla}$ scattering under $\chi=0^{\circ}$ is seen to be modified by the change in $b_{t}{ }^{\prime} / \delta^{\prime}$ upon stretching.

In Figures 9-12, the results of the calculated $H_{\mathrm{V}}$ and $V_{\mathrm{V}}$ patterns under $\chi=0^{\circ}$ are shown in which, as already noted, the effect of birefringence on the patterns is negligible. In these calculations $L / \lambda^{\prime}$ is set to 40 .

Figure 9 shows the effect of stretching on the $H_{\mathrm{V}}$ pattern for $\omega=45^{\circ}$. With increasing elongation ratios $\lambda_{1}$, the equatorial lobes become sharp and thin, while the meridional lobes become diffuse and thick. The tendency agrees well with that of the experimental patterns as 
shown in Figures 1, 2, 3, and 6. At a high elongation ratio $\lambda_{1}=2.0$, the meridional lobes degenerate to the equatorial lobes which are

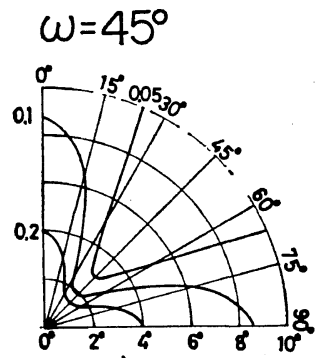

(a) $\lambda_{1}=1.0$

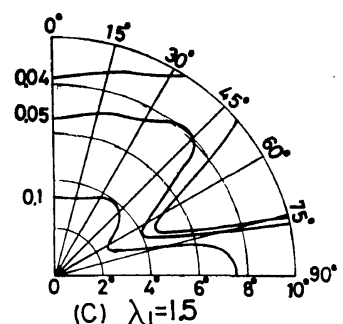

(C) $\lambda_{1}=15$

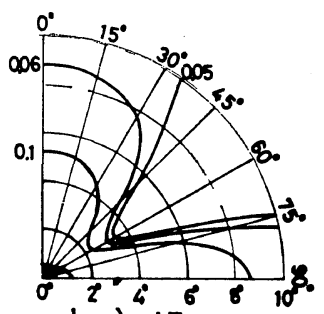

(b) $\lambda_{1}=1.3$

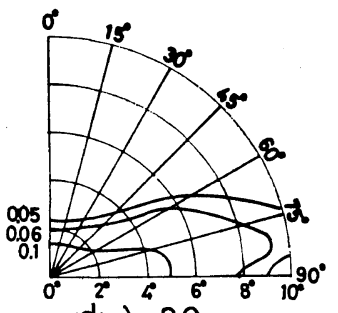

(d) $\lambda_{1}=2.0$
Figure 9. Calculated $H_{\mathrm{V}}$ patterns for $\omega=45^{\circ}$ and $\chi=0^{\circ}$ as a function of $\lambda_{1}$, (a) 1.0 , (b) 1.3 , (c) 1.5 , and (d) 2.0. slightly split. Such a tendency at high elongations is seen in the poly(tetrafluoroethylene) films studied by Rhodes and Stein, ${ }^{12}$ but not in the hydroxypropylcellulose films ${ }^{20}$ (Figure 3) and poly(chlorotrifluoroethylene) films (Figure 2).

The change in the calculated $H_{\mathrm{V}}$ patterns upon initial stretching may be qualitatively understood as follows. In the unstretched state, the rods oriented in meridional and in equatorial directions would contribute most to the $H_{V}$ scattering, since they have maximum induced dipolemoments, $(\boldsymbol{M} \cdot \mathbf{O})_{H_{\mathrm{V}}}$. When the specimen is stretched, the rods in meriodional direction are deformed and elongated as seen in eq 8, so that the corresponding scattering which appears in the equator may become sharp. At the same time, the number of rods in the meridional direction increases, so that the intensity in the equatorial lobes increases. On the other hand the rods in the equatorial direction become shorter, and the population of rods in that direction decreases, so that the corresponding scattering in the meridional direction becomes diffuse and less intense.

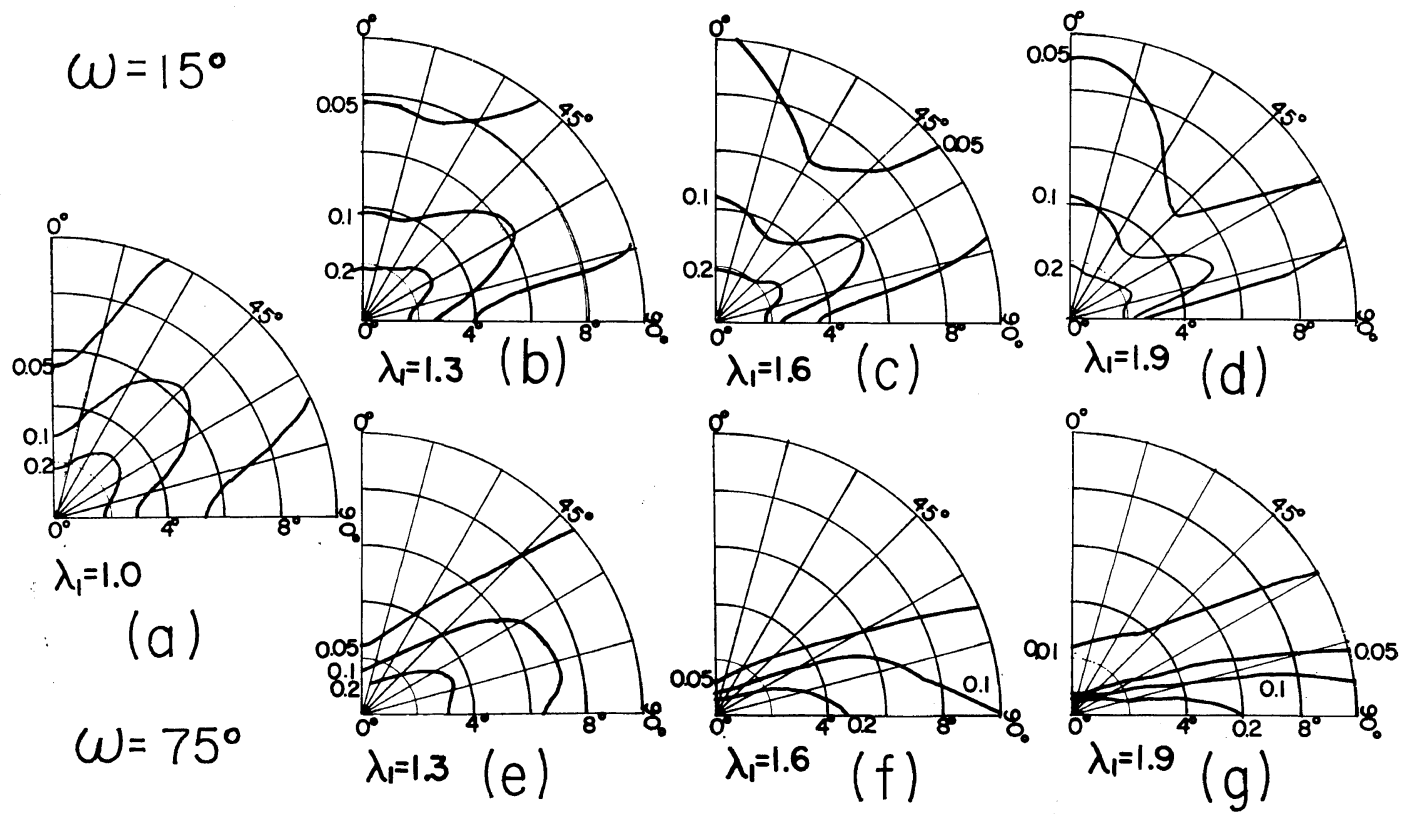

Figure 10. Calculated $H_{\mathrm{V}}$ patterns for $\omega=15^{\circ}$ and $\chi=0^{\circ}$ as a function of $\lambda_{1}$, (a) 1.0, (b) 1.3, (c) 1.6, and (d) 1.9, and for $\omega=75^{\circ}$ and $\chi=0^{\circ}$ as a function of $\lambda_{1}$, (a) 1.0, (e) 1.3, (f) 1.6, and (g) 1.9. 
Light-Scattering Patterns from Stretched Films

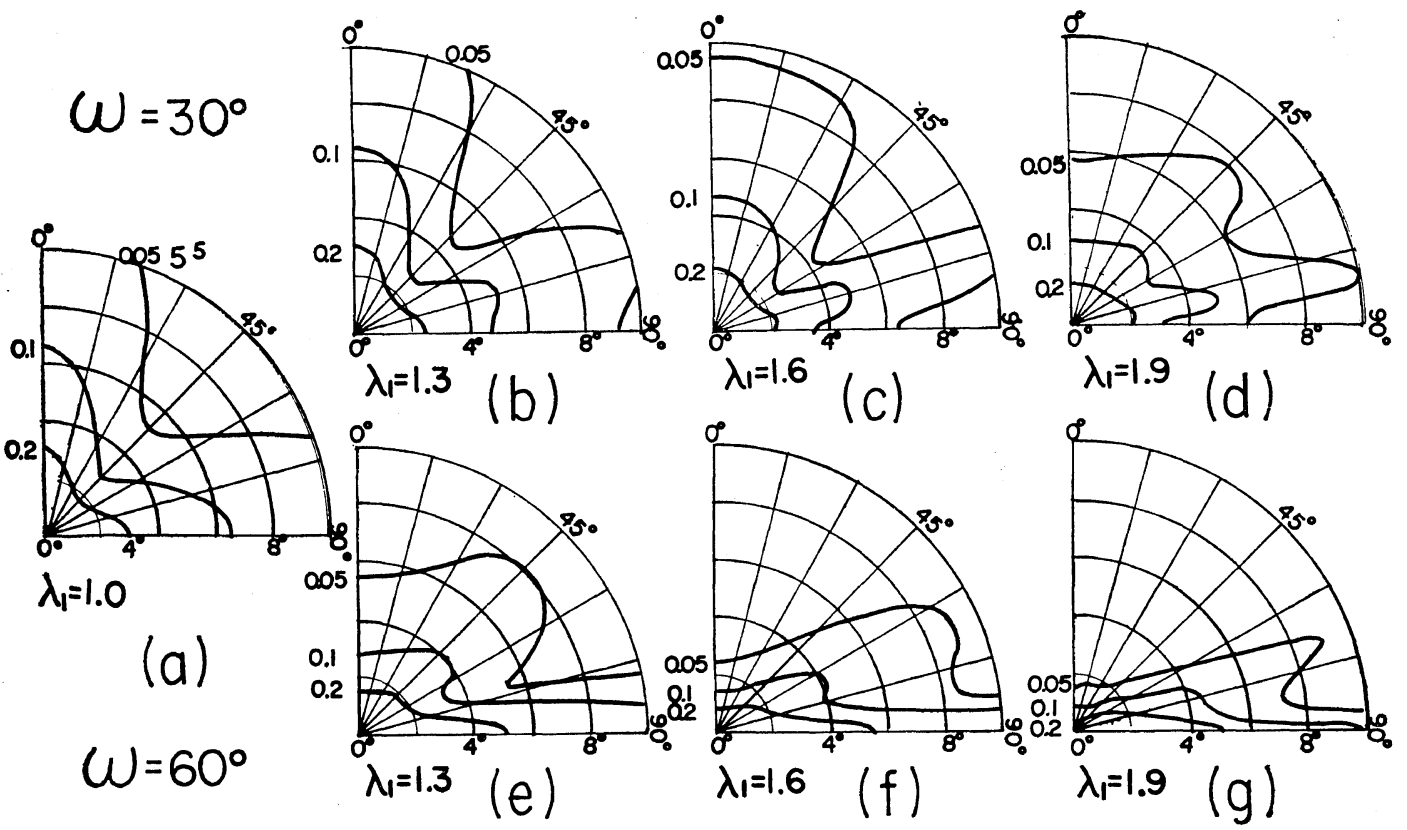

Figure 11. Calculated $H_{\mathrm{V}}$ patterns for $\omega=30^{\circ}$ and $\chi=0^{\circ}$ as a function of $\lambda_{1}$, (a) 1.0 , (b) 1.3, (c) 1.6, and (d) 1.9, and for $\omega=60^{\circ}$ and $\chi=0^{\circ}$ as a function of $\lambda_{1}$, (a) 1.0, (e) 1.3, (f) 1.6, and (g) 1.9.

$$
p=-1 / 3 \quad \omega=45^{\circ}
$$

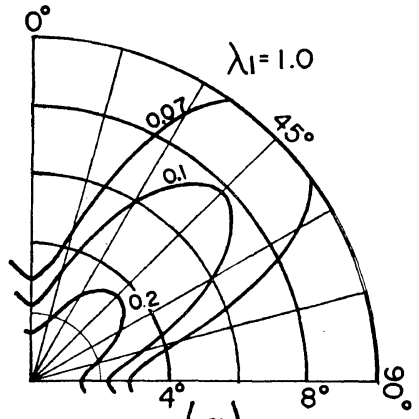

(a)

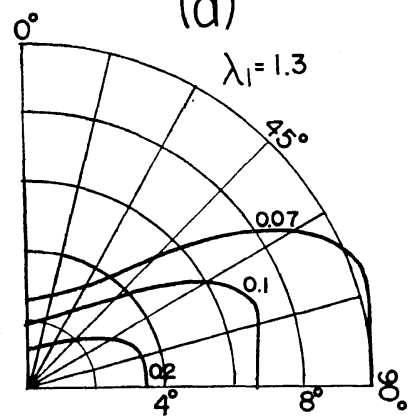

(d)

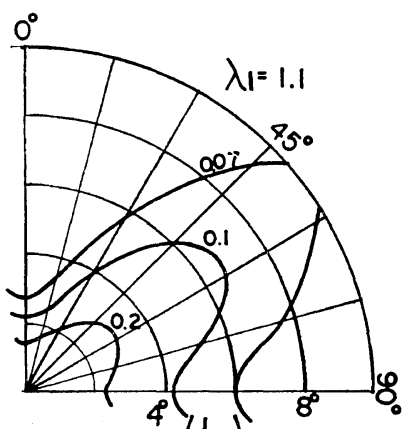

(b)

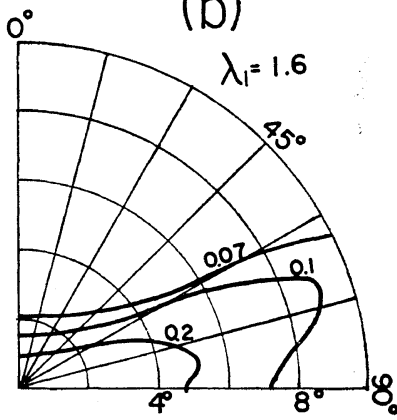

(e)

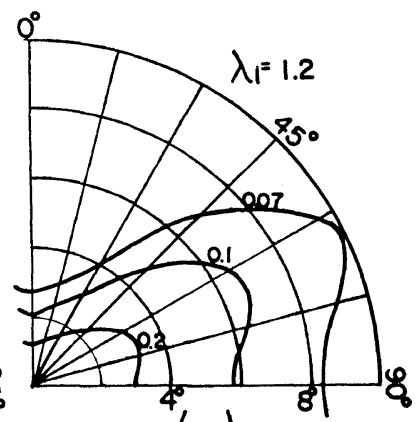

(c)

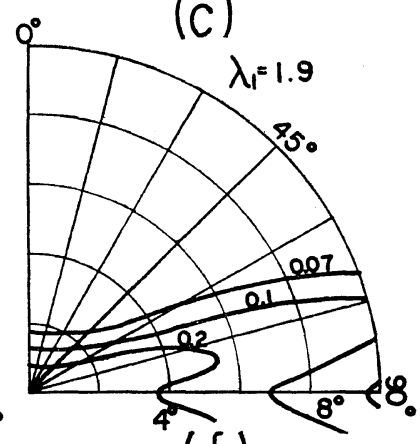

(f)

Figure 12. Calculated $V_{\nabla}$ patterns for $\omega=45^{\circ}, \chi=0^{\circ}$, and $p=-1 / 3$ as a function of $\lambda_{1}$, (a) 1.0 , (b) 1.1 , (c) 1.2 , (d) 1.3 , (e) 1.6 , and (f) 1.9 . 
Similar calculations were carried out by Rhodes and Stein ${ }^{12}$ based on the model in which (i) the length of the rods remain constant at $L$ through the deformation process, (ii) the distribution function of $N^{\prime}\left(\alpha^{\prime}\right)$ is given by

$$
N^{\prime}\left(\alpha^{\prime}\right)=N_{0}\left[\lambda_{1}^{-2} \cos ^{2} \alpha^{\prime}+\lambda_{1}^{2} \sin ^{2} \alpha^{\prime}\right]^{-1 / 2}
$$

which is somewhat similar to $N^{\prime}\left(\alpha^{\prime}\right)$ given by eq 9 , and (iii) $\omega^{\prime}$ is given by an empirical function of

$$
\omega^{\prime}=\exp \left\{-\kappa\left[N^{\prime}\left(\alpha^{\prime}\right)-1\right]\right\}
$$

Where $\kappa$ is a parameter related to ease of optical axis orientation within the rod. The result of the calculations was not as satisfactory as that reported here in describing the change of the meridional and equatorial lobes of the patterns upon stretching.

Calculations were carried out also by Samuels $^{20}$ on the basis of a similar model. The model is based on the assumptions: (i) as in the model of Rhodes and Stein, the length of the rods is constant through the deformation, (ii) $N^{\prime}\left(\alpha^{\prime}\right)$ is given by either eq 47 or

$$
N^{\prime}\left(\alpha^{\prime}\right)=N_{0}\left[\lambda_{1}{ }^{2} \cos ^{2} \alpha^{\prime}+\lambda_{1}{ }^{-2} \sin ^{2} \alpha^{\prime}\right]^{1 / 2}
$$

and (iii) $\omega^{\prime}$ is independent of $\alpha^{\prime}$ and is an adjusting parameter varied upon stretching. Again the result is not as satisfactory in describing the change of the lobes. However the model of Samuels can better explain the patterns at high elongations, especially the splitting of the meridional lobes of $H_{\mathrm{V}}$ patterns as shown in Figure $3 \mathrm{e}$ and $3 \mathrm{f}$, which cannot be explained in terms of the model reported here. This latter, which assumes an affine deformation, is usually good only for the initial stage of the elongations.

Figures 10 and 11 show the effect of stretching on $H_{\mathrm{V}}$ patterns for other values of $\omega$. Although the $H_{\mathrm{V}}$ patterns for $\omega=15^{\circ}$ and $75^{\circ}$ are identical in the unstretched state (Figure 10a), there appears to be a difference between the two cases when the specimen is stretched, which might be used to distinguish between the two cases. The same phenomenum can be seen for the $H_{\mathrm{V}}$ patterns for $\omega=30^{\circ}$ and $60^{\circ}$ as shown in Figure 11.

By comparing Figures 9, i0, and 11 with the experimental patterns, it may be seen that the case of where the value of $\omega$ about $45^{\circ}$ can best explain the experimental scattering patterns.

Figure 12 shows the calculated $V_{\mathrm{V}}$ patterns for $\omega=45^{\circ}$ and $p=b_{\mathrm{t}}{ }^{\prime} / \delta^{\prime}=-1 / 3$. If $\left\langle\alpha^{\prime}\right\rangle_{\mathrm{av}}$ is the average polarizability of the scattering element, $b_{\mathrm{t}}{ }^{\prime}$ is given by $b_{\mathrm{t}}{ }^{\prime}=\left\langle\alpha^{\prime}\right\rangle_{\mathrm{av}}-\alpha_{\mathrm{s}}{ }^{\prime}-\left(\delta^{\prime} / 3\right)$ where $\alpha_{\mathrm{s}}{ }^{\prime}$ is the polarizability of the surrounding medium, so that $p$ is given by $p=\left(\left\langle\alpha^{\prime}\right\rangle_{\mathrm{av}}-\right.$ $\left.\alpha_{\mathrm{s}}{ }^{\prime}\right) / \delta^{\prime}-1 / 3$. Consequently the case where $p=$ $-1 / 3$ corresponds to the case where $\left\langle\alpha^{\prime}\right\rangle_{\mathrm{av}}=\alpha_{\mathrm{s}}{ }^{\prime}$, i.e., the case where the density contribution to $V_{\mathrm{V}}$ scattering is very small. The $V_{\mathrm{V}}$ pattern for the unstretched state (Figure 12a) has a four-fold symmetrical intensity distribution with maximum intensity at $\mu=45^{\circ}$. Upon stretching, the lobes are oriented toward the equator and the intensity at the equator increases. The splitting of the lobes at the equator becomes less clear at initial elongations up to $30 \%$. Upon further stretching, the splitting again becomes clear.

By comparing Figure 12 with Figure 4 it may be seen that the calculated results are qualitatively in agreement with the experimental results except for the deviation at the initial elongations where the calculated results show less splitting of the lobes compared to those of the experimental patterns. The deviation might be attributed to the assumption that $p$ is independent of $\alpha^{\prime}$. The sharp splitting of the experimental lobes around $30 \%$ could not be accounted for by simply varying the value of $p$, but was accounted for by introducing the angular dependence of $p$. The angular dependence may result partially an angular dependence of $\left\langle\alpha^{\prime}\right\rangle_{\mathrm{av}}$ which results from the deformation of the rods in terms of their size.

Similar calculations were carried out on some other models which may be considered by changing the orientation and deformation behaviors of the rod as a whole and by changing the orientation behavior of the principal optical axis of the scattering element;

I. As for the deformation and orientation of the rod as a whole, two models were considered.

(I-1) Both deformation and orientation of the rod occur affinely.

(I-2) The length of the rod is kept constant, 
Light-Scattering Patterns from Stretched Films

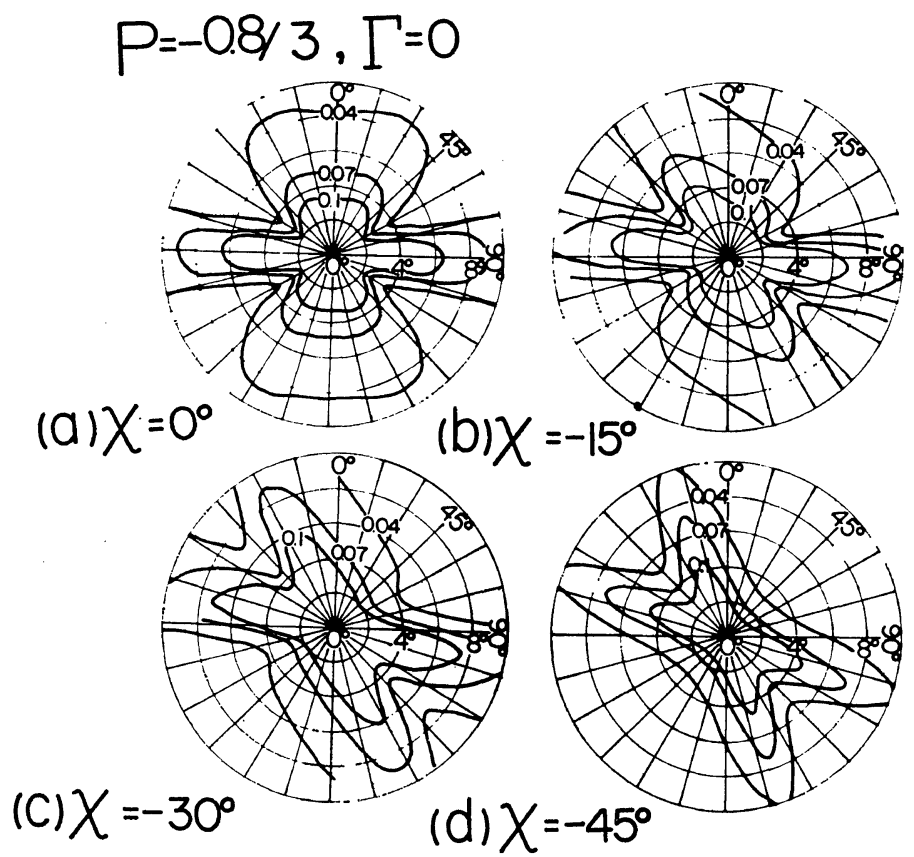

Figure 13. Calculated $H_{\mathrm{V}}$ patterns for $\lambda_{1}=1.4$ and $\Gamma=0$ as a function of $\chi$, (a) $0^{\circ}$, (b) $-15^{\circ}$, (c) $-30^{\circ}$, and (d) $-45^{\circ}$.

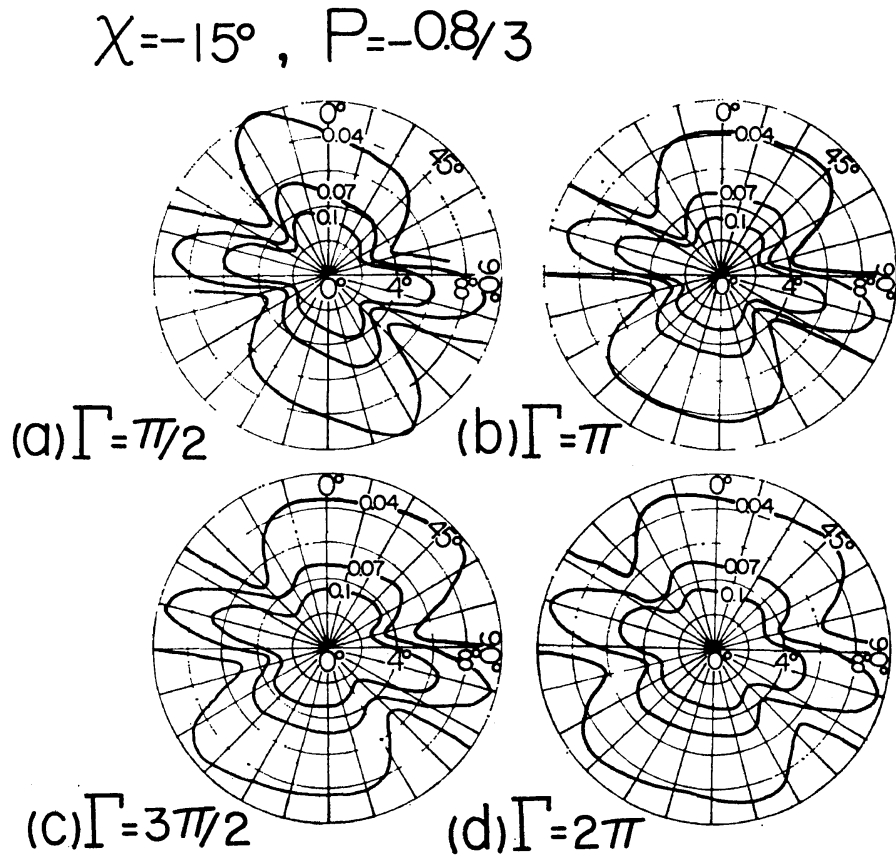

Figure 14. Calculated $H_{\mathrm{V}}$ patterns for $\lambda_{1}=1.4$ and $\chi=-15^{\circ}$ as a function of $\Gamma$, (a) $\pi / 2$, (b) $\pi$, (c) $3 \pi / 2$, and (d) $2 \pi$. 


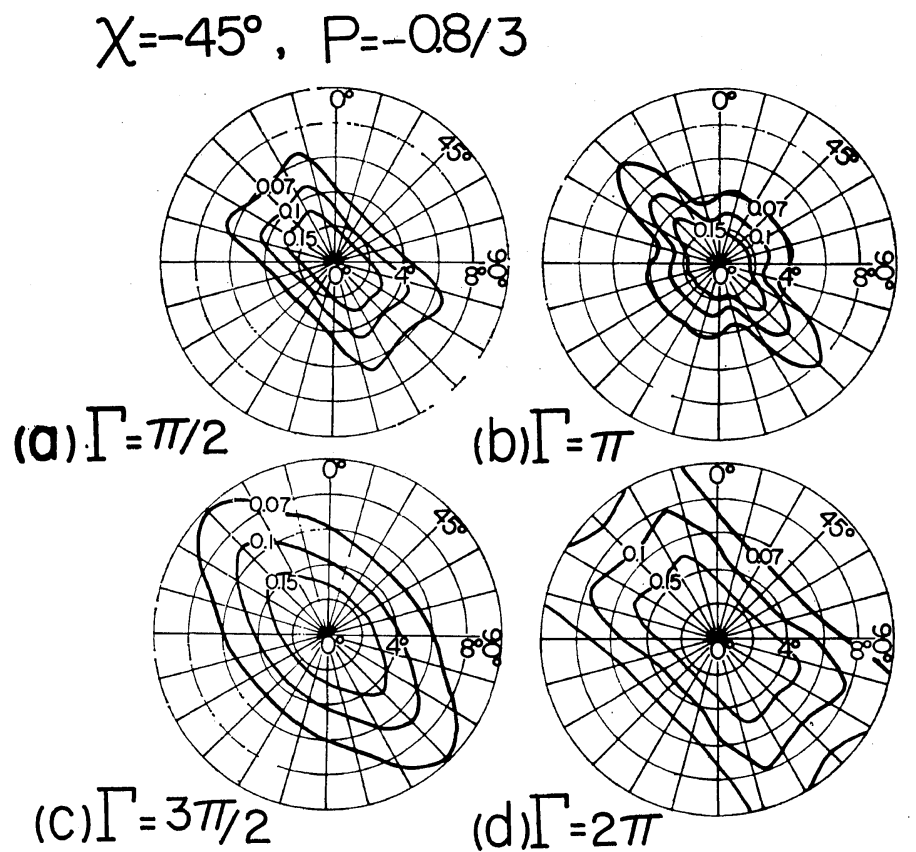

Figure 15. Calculated $H_{\mathrm{V}}$ patterns for $\lambda_{1}=1.4$ and $\chi=-45^{\circ}$ as a function of $\Gamma$, (a) $\pi / 2$, (b) $\pi$, (c) $3 \pi / 2$, and (d) $2 \pi$.

but only its orientation changes affinely.

II. As for the orientation of the optic axis, three models were considered.

(II-1) Affine orientation

(II-2) $\omega^{\prime}=\omega \exp \left[-\kappa\left(\lambda_{1}-\lambda_{1}^{-1}\right)\right]$

(II-3) $\omega^{\prime}=\omega \exp \left[-\kappa\left(\lambda_{1}-\lambda_{1}^{-1}\right) \cos ^{2} \alpha^{\prime}\right]$

Calculations based on the six possible models from the combinations of (I-i) and (II- $j$ ) were attempted and the results seemed to indicate that the model given by the set of (I-1) and (II-1), i.e., the model described earlier was best at explaining the characteristic change of the experimental $H_{V}$ and $V_{\mathrm{V}}$ scattering patterns at the initial stage of stretching.

In Figures 13 to 15, calculated results are shown for the effect of birefringence on the patterns under crossed polarizers as a function of the angle $\chi$ and retardation $\Gamma$. The calculations were carried out for a case of the local elongation ratio of $\lambda_{1}=1.4$ at which the calculated $H_{\mathrm{V}}$ pattern (see Figure 13a) fits the experimental $H_{\mathrm{V}}$ pattern from the stretched collagen films (see Figure 5c). In the calculations it was assumed that $R_{1}$ is nearly equal to $R_{2}$ in eq 31 .
Figure 13 shows a change in the pattern under crossed polarizers on rotation of the stretching direction for a case of $\Gamma=0$, i.e., a fictitious case where there is no effect of retardation on the scattering.

The case of $\chi=0^{\circ}$ (Figure 13a) is identical to that of the $H_{\mathrm{V}}$ scattering discussed before. The change of the patterns upon rotating the stretching direction is interpreted as resulting from the fact that the rods contributing primarily to the scattering are different for different values of $\chi$.

In Figures 14 and 15, the effect of birefringence on the scattering patterns are shown for $\chi=-15$ and $-45^{\circ}$, respectively, as a function of retardation $\Gamma$. As seen in eq 30 , the scattering under the crossed polarizers depends also on $p$ when the specimen is birefringent, and $\chi$ is not equal to 0 or $90^{\circ}$. The value of $p$ was chosen as $-0.8 / 3$ in Figures 14 and 15 and was determined by fitting the theoretical and experimental $V_{\mathrm{V}}$ patterns for the stretched films under $\chi=0^{\circ}$. As seen in the figures, the scattering patterns are significantly modified with increasing retardation. The experimental pat- 
terns shown in Figures $5 \mathrm{e}$ to $5 \mathrm{~g}$ agree fairly well with the theoretical patterns (Figures 14 and 15) obtained for small value of $\Gamma$.

\section{CONCLUSIONS}

The deformation mechanism of crystalline polymer films having an anisotropic fibrillar, nonspherulitic crystalline superstructure was studied using the polarized light-scattering method. There was found to be a common characteristic change in the $H_{\mathrm{V}}$ patterns observed on stretching for the (i) collagen, (ii) poly(chlorotrifluoroethylene), (iii) water soluble hydroxypropylcellulose, (iv) polypropylene-polyethylene A-B type block copolymer and (v) poly(terafluoroethylene) films in that the meridional lobes of the patterns become diffuse in the scattered intensity distribution, whilst the equatorial lobes become sharp at the initial stage of elongation.

The characteristic change was explained in terms of the affine deformation of the fibrils, and was suggested to arise from (i) the deformation of the fibrils in terms of their size as well as (ii) the orientation of the fibrils as a whole and (iii) the orientation of the optical axes of the scattering elements within the fibrils toward stretching direction.

When the specimen is stretched and birefringent, the effect of retardation on the scattering patterns was shown to be significant when the stretching direction is inclined with respect to the polarization direction of the polarizer or analyzer, but to be negligible when it is set parallel to the polarization directions.

Acknowledgments. The authors wish to express their appreciation to Dr. R. J. Samuels, Hercules Incorporated, Wilmington, Delaware, U.S.A. for his offer of the excellent $H_{\mathrm{V}}$ and $V_{\mathrm{V}}$ patterns of the hydroxypropylcellulose films in Figures 3 and 4 . The authors also wish to acknowledge the assistance of the Research Laboratory, Japan Leather Co., Ltd, where the test films of collagen were prepared.

\section{REFERENCES}

1. R. S. Stein and P. R. Wilson, J. Appl. Phys., 33, 1914 (1962).
2. R. S. Stein and M. B. Rhodes, J. Appl. Phys., 31, 1873 (1960).

3. R. J. Samuels, J. Polym. Sci., Part A-2, 9, 2165 (1971).

4. M. Moritani, N. Hayashi, A. Utsuo, and H. Kawai, Polymer J. (Japan), 2, 74 (1971).

5. J. Borch, R. Muggli, A. Sarko, and R. H. Marchessault, J. Appl. Phys., 42, 4570 (1971).

6. M. B. Rhodes and R. S. Stein, ibid., 39, 4903 (1968).

7. S. B. Clough, J. J. van Aartsen, and R.S. Stein, ibid., 36, 3072 (1965).

8. T. Hashimoto, A. Todo, and H. Kawai, J. Polym. Sci., Polymer Phys., Ed., 11, 149 (1973).

9. J. J. van Aartsen and R. S. Stein, ibid., Part $A-2,9,295$ (1971).

10. R. J. Samuels, ibid., Part C, 13, 37 (1966).

11. T. Hashimoto, Y. Murakami, N. Hayashi, and H. Kawai, to be submitted.

12. M. B. Rhodes and R.S. Stein, J. Polymer Sci., Part A-2, 7, 1539 (1969).

13. G. C. Adams and R.S. Stein, ibid., Part $A-2$, 6, 31 (1968).

14. J. Borsch and R. H. Marchessanlt, ibid., Part C, 28, 153 (1969).

15. J. C. W. Chien and E. P. Chang, Macromolecules, 5, 610 (1973).

16. P. H. Geil, "Polymer Single Crystals," Interscience Publishers, Inc., New York, N.Y., 1963.

17. M. Matsuo, S. Nomura, T. Hashimoto, and H. Kawai, to be published, Rept. Prog. Polymer Phys. Japan, 16, in press.

18. J. J. van Aartsen, Eur. Polym. J., 6, 1095 (1970).

19. N. Hayashi and H. Kawai, Polymer J. 3, 140 (1972).

20. R. J. Samuels, J. Polym. Sci., Part A-2, 7, 1197 (1969).

21. A. Asanuma, a part of M.S. Thesis presented at the Department of Polymer Chemistry, Faculty of Engineering, Kyoto University, Kyoto, on March, 1970.

22. T. Hashimoto, N. Hayashi, Y. Murakami, and H. Kawai, to be submitted to Polymer J., Rept. Prog. Polymer Phys. Japan, 16, in press.

23. A. E. M. Keijizers, J. J. van Aartsen, and W. Prins, J. Amer. Chem. Soc., 90, 3167 (1968).

24. C. J. Speerschneider and C. H. Li, J. Appl. Phys., 33, 1871 (1962).

25. M. Motegi, M. Moritani and H. Kawai, J. Polym. Sci., Part A-2, 8, 499 (1970).

26. W. Chu and R. S. Stein, ibid., Part A-2, 8, 489 (1970).

27. Y. Murakami, N. Hayashi, T. Hashimoto, and H. Kawai, Polymer J., 4 (1973) in press. 